\title{
The K-theory of Heegaard quantum lens spaces
}

\author{
Piotr M. Hajac, Adam Rennie, and Bartosz Zieliński \\ Dedicated to Alan Carey on the occasion of his 60th birthday
}

\begin{abstract}
Representing $\mathbb{Z} / N \mathbb{Z}$ as roots of unity, we restrict a natural $U(1)$-action on the Heegaard quantum sphere to $\mathbb{Z} / N \mathbb{Z}$, and call the quotient spaces Heegaard quantum lens spaces. Then we use this representation of $\mathbb{Z} / N \mathbb{Z}$ to construct an associated complex line bundle. This paper proves the stable non-triviality of these line bundles over any of the quantum lens spaces we consider. We use the pullback structure of the $C^{*}$-algebra of the lens space to compute its $K$-theory via the Mayer-Vietoris sequence, and an explicit form of the odd-to-even connecting homomorphism to prove the stable non-triviality of the bundles. On the algebraic side we prove the universality of the coordinate algebra of such a lens space for a particular set of generators and relations. We also prove the non-existence of non-trivial invertibles in the coordinate algebra of a lens space. Finally, we prolongate the $\mathbb{Z} / N \mathbb{Z}$-fibres of the Heegaard quantum sphere to $U(1)$, and determine the algebraic structure of such a $U(1)$-prolongation.
\end{abstract}

Mathematics Subject Classification (2010). 46L80, 46L85.

Keywords. Principal actions on $\mathrm{C}^{*}$-algebras, strong connections, Milnor idempotent formula for the Mayer-Vietoris 6-term exact sequence, generators of $K$-groups.

\section{Introduction and preliminaries}

0.1. Introduction. It is hard to deny that lens spaces are interesting. Indeed, they have provided a rich source of examples highlighting subtle phenomena in topology. They are simple examples of closed 3-manifolds not determined by their homology and fundamental group alone. They also give examples of spaces that might be homotopic but not homeomorphic. Even today they still provide a fertile arena in which to study topological questions, e.g., see [17].

A typical feature of lens spaces is that they possess non-trivial line bundles giving rise to torsion in $K$-theory. This property of lens spaces remains a characteristic feature of their quantum analogues, and is a focal point of this paper. In brief, we choose a particular family of quantum lens spaces, define natural complex line bundles over them, and prove that they generate torsion in the $K_{0}$-group.

More precisely, we study a family of three-dimensional lens spaces arising from a particular family of quantum 3-spheres, namely the Heegaard quantum spheres 
$S_{p q \theta}^{3}$ [2]. The $C^{*}$-algebras of these Heegaard quantum lens spaces were defined in [13] as fixed-point subalgebras for a $\mathbb{Z} / N \mathbb{Z}$-action obtained by restricting the natural (diagonal) $U(1)$-action $\alpha$ on the $C^{*}$-algebra $C\left(S_{p q \theta}^{3}\right)$ of the Heegaard quantum sphere. Likewise, we consider fixed-point subalgebras of the coordinate algebra $\mathcal{O}\left(S_{p q \theta}^{3}\right)$ of the Heegaard quantum sphere. We denote the thus obtained coordinate algebras and $C^{*}$-algebras of these quantum lens spaces by $\mathcal{O}\left(L_{p q \theta}^{N}\right)$ and $C\left(L_{p q \theta}^{N}\right)$ respectively.

Note that in [19] a different $U(1)$-action was used to define another class of Heegaard quantum lens spaces. Both of these types of Heegaard quantum lens spaces are different from those quantum lens spaces studied in [14]. The latter are graph $C^{*}$-algebras and the former are pullback $C^{*}$-algebras. This is a crucial technical difference between these two families of $C^{*}$-algebras resulting in application of different tools to study their $K$-theory.

Next, we again represent $\mathbb{Z} / N \mathbb{Z}$ via roots of unity and define the following associated module

$$
L_{N}:=\left\{x \in C\left(S_{p q \theta}^{3}\right) \mid \alpha_{e^{\frac{2 \pi i}{N}}}(x)=e^{\frac{2 \pi i}{N}} x\right\} \subseteq C\left(S_{p q \theta}^{3}\right)
$$

over $C\left(L_{p q \theta}^{N}\right)$. This is a finitely generated projective module defining a natural complex line bundle for each of our quantum lens spaces. Our main result can be now summarized as follows.

Theorem 0.1. The left $C\left(L_{p q \theta}^{N}\right)$-module $L_{N}$ is not stably free, and $\left[L_{N}\right]-\left[C\left(L_{p q \theta}^{N}\right)\right]$ generates the torsion part of $K_{0}\left(C\left(L_{p q \theta}^{N}\right)\right)$.

On the way, we prove that $\mathcal{O}\left(L_{p q \theta}^{N}\right)$ is universal for a certain set of generators and relations. Having done this, we show that $\mathcal{O}\left(S_{p q \theta}^{3}\right)$ contains no invertibles other than non-zero multiples of the identity. This allows us to prove that the $\mathcal{O}(\mathbb{Z} / N \mathbb{Z})$-comodule algebra $\mathcal{O}\left(S_{p q \theta}^{3}\right)$ is non-cleft, which reflects the non-triviality of the noncommutative $\mathbb{Z} / N \mathbb{Z}$-principal bundle $S_{p q \theta}^{3} \rightarrow L_{p q \theta}^{N}$. However, to conclude a stronger result that the finitely generated projective $\mathcal{O}\left(L_{p q \theta}^{N}\right)$-module

$$
\mathscr{L}_{N}:=\left\{x \in \mathcal{O}\left(S_{p q \theta}^{3}\right) \mid \alpha_{e^{\frac{2 \pi i}{N}}}(x)=e^{\frac{2 \pi i}{N}} x\right\} \subseteq \mathcal{O}\left(S_{p q \theta}^{3}\right)
$$

is not stably free, we turn to $C^{*}$-algebras. Representing $L_{N}$ by an idempotent with entries in $\mathcal{O}\left(L_{p q \theta}^{N}\right)$, we infer the stable non-triviality of $\mathscr{L}_{N}$ over $\mathcal{O}\left(S_{p q \theta}^{3}\right)$ from our main result.

The final section proves a quantum version of the classical phenomenon that $S^{3} \times_{\mathbb{Z} / N \mathbb{Z}} U(1) \cong S^{3} \times U(1)$, in both the algebraic and $C^{*}$-setting. Namely, we prove that

$$
\begin{aligned}
& C\left(S_{p q \theta}^{3}\right) \bar{\otimes} C(U(1)) \cong\left(C\left(S_{p q \theta}^{3}\right) \bar{\otimes} C(U(1))\right)^{\mathbb{Z} / N \mathbb{Z}}, \\
& \mathcal{O}\left(S_{p q \theta}^{3}\right) \otimes \mathcal{O}(U(1)) \cong\left(\mathcal{O}\left(S_{p q \theta}^{3}\right) \otimes \mathcal{O}(U(1))\right)^{\mathbb{Z} / N \mathbb{Z} .}
\end{aligned}
$$


Here and in what follows, the unadorned tensor product stands for the algebraic tensor product over the ground field $k$, typically of complex numbers. Since in this paper there is no ambiguity concerning $C^{*}$-completions of the algebraic tensor product, we simply use $\bar{\otimes}$ to denote the completed tensor product. Also, we use the convention that, for algebras $A, B$, and coalgebras $C, D$, the symbol ${ }_{A}^{C} \operatorname{Hom}_{B}^{D}$ signifies the set of $k$-linear homomorphisms that are left $A$-linear, right $B$-linear, left $C$-colinear and right $D$-colinear.

0.2. Principal comodule algebras. The comultiplication, counit and the antipode of a Hopf algebra $H$ are denoted by $\Delta, \varepsilon$ and $S$, respectively. A right $H$-comodule algebra $P$ is a unital associative algebra equipped with an $H$-coaction $\Delta_{P}: P \rightarrow P \otimes H$ that is an algebra map. For a comodule algebra $P$, we call

$$
P^{\text {co } H}:=\left\{p \in P \mid \Delta_{P}(p)=p \otimes 1\right\}
$$

the subalgebra of coaction-invariant elements in $P$. A left coaction on $V$ is denoted by ${ }_{V} \Delta$. For comultiplications and coactions, we often employ the HeynemannSweedler notation with the summation symbol suppressed:

$$
\Delta(h)=: h_{(1)} \otimes h_{(2)}, \quad \Delta_{P}(p)=: p_{(0)} \otimes p_{(1)}, \quad V \Delta(v)=: v_{(-1)} \otimes v_{(0)} .
$$

With this notation, the convolution product of maps $f$ and $g$ from a coalgebra to an algebra is given by $(f * g)(h):=f\left(h_{(1)}\right) g\left(h_{(2)}\right)$.

If $M$ is a right comodule over a coalgebra $C$ and $N$ is a left $C$-comodule, then we define their cotensor product as

$$
M \underset{C}{\square} N:=\left\{t \in M \otimes N \mid\left(\Delta_{M} \otimes \mathrm{id}\right)(t)=\left(\mathrm{id} \otimes{ }_{N} \Delta\right)(t)\right\} .
$$

In particular, for a right $H$-comodule algebra $P$ and a left $H$-comodule $V$, we observe that $P \square_{H} V$ is a left $P^{\text {co } H}$-module in a natural way. Furthermore, if $V$ is a Hopf algebra with comultiplication $\tilde{\Delta}$, a Hopf algebra surjection $\pi: V \rightarrow H$, and a left coaction $_{V} \Delta:=(\pi \otimes$ id $) \circ \tilde{\Delta}$, then $P \square_{H} V$ becomes a $V$-comodule algebra for the coaction id $\otimes \tilde{\Delta}$.

An $H$-comodule algebra $P$ is called principal [3] if:

(1) $P \otimes_{B} P \ni p \otimes q \mapsto \operatorname{can}(p \otimes q):=p q_{(0)} \otimes q_{(1)} \in P \otimes H$ is bijective;

(2) $\exists s \in{ }_{B} \operatorname{Hom}^{H}(P, B \otimes P): m \circ s=$ id, where $m$ is the multiplication map;

(3) the antipode of $H$ is bijective.

Here (1) is the Hopf-Galois (freeness) condition, (2) means equivariant projectivity of $P$, and (3) ensures a left-right symmetry of the definition (everything can be re-written for left comodule algebras). The inverse of the map can can be written explicitly using Heynemann-Sweedler like notation: $\operatorname{can}^{-1}(p \otimes h)=p h^{[1]} \otimes_{B} h^{[2]}$. Here the map

$$
H \ni h \longmapsto \operatorname{can}^{-1}(1 \otimes h)=: h^{[1]} \underset{B}{\otimes} h^{[2]} \in P \underset{B}{\otimes} P
$$


is called a translation map, and satisfies $h^{[1]} h^{[2]}=\varepsilon(h)$.

One of the key properties of principal comodule algebras is that, for any finitedimensional left $H$-comodule $V$, the left $P^{\text {co } H}$-module $P \square_{H} V$ is finitely generated projective [3]. Here $P$ plays the role of a principal bundle and $P \square_{H} V$ plays the role of an associated vector bundle. Therefore, we call $P \square_{H} V$ an associated module. On the other hand, if $V$ is a Hopf algebra and $P \square_{H} V$ is a $V$-comodule algebra as described above, then (under some minor technical assumptions) the principality of the $H$-coaction on $P$ implies the principality of the $V$-coaction on $P \square_{H} V$ (see [12]). We call the principal comodule algebra $P \square_{H} V$ the $V$-prolongation of $P$ because it is a direct analogue of a prolongation of a principal bundle that is obtained by enlarging its structure group.

If $H$ is a Hopf algebra with bijective antipode and $P$ is a right $H$-comodule algebra, then one can show (cf. [3]) that it is principal if and only if there exists a linear map

$$
\ell: H \longrightarrow P \otimes P, \quad h \longmapsto \ell(h)=: \ell(h)^{\langle 1\rangle} \otimes \ell(h)^{\langle 2\rangle},
$$

such that, for all $h \in H, \ell$ satisfies the three equations

$$
\begin{gathered}
\ell(h)^{\langle 1\rangle} \ell(h)^{\langle 2\rangle}(0) \otimes \ell(h)^{\langle 2\rangle}{ }_{(1)}=1 \otimes h, \\
S\left(h_{(1)}\right) \otimes \ell\left(h_{(2)}\right)^{\langle 1\rangle} \otimes \ell\left(h_{(2)}\right)^{\langle 2\rangle}=\ell(h)^{\langle 1\rangle}{ }_{(1)} \otimes \ell(h)^{\langle 1\rangle}(0) \otimes \ell(h)^{\langle 2\rangle}, \\
\ell\left(h_{(1)}\right)^{\langle 1\rangle} \otimes \ell\left(h_{(1)}\right)^{\langle 2\rangle} \otimes h_{(2)}=\ell(h)^{\langle 1\rangle} \otimes \ell(h)^{\langle 2\rangle}{ }_{(0)} \otimes \ell(h)^{\langle 2\rangle}{ }_{(1)} .
\end{gathered}
$$

Any such map $\ell$ can be made unital [3]. It is then called a strong connection [8, 5, 3], and can be thought of as an appropriate lifting of the translation map. Given a strong connection, we can explicitly compute an idempotent representing the module $P \square_{H} V$ [3]. In particular, if $\operatorname{dim} V=1$, the coaction ${ }_{V} \Delta$ is determined by a grouplike $g \in H$, i.e., $V \Delta(1)=g \otimes 1$. Then, in order to obtain an idempotent representing $P \square_{H} V$, we write $\ell(g)=\sum_{i} x_{i} \otimes e_{i}$, where elements $e_{i}$ are chosen to be linearly independent. The desired idempotent matrix is given by $e_{i j}:=e_{i} x_{j}$.

A special class of principal comodule algebras is distinguished by the existence of a cleaving map. A cleaving map is defined as a unital right $H$-colinear convolutioninvertible map $j: H \rightarrow P$. Having a cleaving map, one can define a strong connection as $\ell:=\left(j^{-1} \otimes j\right) \circ \Delta$, where $j^{-1}$ stands for the convolution inverse of $j$. Comodule algebras admitting a cleaving map are called cleft. In particular, if $j$ is a colinear algebra homomorphism, it is a cleaving map (not the other way round). In this special case a cleaving map serves as an analogue of a trivialisation of a principal bundle. Therefore, we can refer to comodule algebras admitting a cleaving map that is an algebra homomorphism as trivial comodule algebras. Note that proving the non-cleftness of a principal comodule algebra is stronger than proving its non-triviality.

All modules associated with cleft comodule algebras are always free. Also, one can show that a cleaving map is automatically injective. Therefore, as the value of a 
cleaving map on a group-like element is invertible, we can conclude that the existence of a non-trivial group-like in $H$ necessitates the existence of an invertible element in $P$ that is not a multiple of 1 . Hence one of the ways to prove the non-cleftness of a principal comodule algebra over a Hopf algebra with a non-trivial group-like is to show the lack of non-trivial invertibles in the comodule algebra.

\subsection{From quantum disc to quantum lens spaces}

0.3.1. Quantum disc. A two-parameter family of quantum unit discs was defined in [15]. Here we consider the one parameter subfamily studied therein. We start with a coordinate $*$-algebra $\mathcal{O}\left(D_{p}\right)$ generated by a single element $x$ and the relation

$$
x^{*} x-p x x^{*}=1-p, \quad 0 \leq p<1 .
$$

We can introduce another algebra $\mathcal{O}^{-}\left(D_{p}\right)$ generated by $x_{-}$with relation

$$
x_{-}^{*} x_{-}-p^{-1} x_{-} x_{-}^{*}=1-p^{-1} .
$$

Then assignment $x \mapsto x_{-}^{*}$ can be extended to a $*$-algebra isomorphism

$$
\kappa_{p}: \mathcal{O}\left(D_{p}\right) \longrightarrow \mathcal{O}^{-}\left(D_{p}\right)
$$

Let us denote for brevity $X:=\left(1-x x^{*}\right)$, so that $1-x^{*} x=p X$. It follows from (0.12) that $X x=p x X$, and more generally

$$
X^{k} x^{n}=p^{k n} x^{n} X^{k}, \quad X^{k} x^{* n}=p^{-k n} x^{* n} X^{k}, \quad n, k \in \mathbb{N},
$$

where the second equation follows from the self-adjointness of $X$. The universal $C^{*}$ algebra for the relation $(0.12)$ contains $\mathcal{O}\left(D_{p}\right)$ and is isomorphic with the Toeplitz algebra $\mathcal{T}$ for all $0 \leq p<1$ [15]. In particular, we can take the relation (0.12) with $p=0$ as a convenient presentation for the $C^{*}$-algebra $\mathcal{T}$. Then $(0.12)$ reduces to $x^{*} x=1$, so that $x$ becomes an isometry.

0.3.2. Heegaard quantum sphere. For $0 \leq p, q, \theta<1, \theta$ irrational, the coordinate algebra of the Heegaard quantum sphere $\mathcal{O}\left(S_{p q \theta}^{3}\right)$ [2] is the universal $*$-algebra generated by two elements $a$ and $b$ satisfying the relations

$$
\begin{gathered}
a b=e^{i 2 \pi \theta} b a, \quad a b^{*}=e^{-i 2 \pi \theta} b^{*} a, \\
a^{*} a-p a a^{*}=1-p, \quad b^{*} b-q b b^{*}=1-q, \\
\left(1-a a^{*}\right)\left(1-b b^{*}\right)=0 .
\end{gathered}
$$

Recall that $\left\{a, a^{*}\right\}$ and $\left\{b, b^{*}\right\}$ generate algebras $\mathcal{O}\left(D_{p}\right)$ and $\mathcal{O}\left(D_{q}\right)$ respectively (see $[2,(2.44)])$. Furthermore, by Subsection 0.3 .1 , for $A:=\left(1-a a^{*}\right)$, $B:=\left(1-b b^{*}\right)$, we have the relations

$$
A a=p a A, \quad A b=b A, \quad B a=a B, \quad B b=q b B, \quad A^{*}=A, \quad B^{*}=B .(0.17)
$$


Now we can write a basis of $\mathcal{O}\left(S_{p q \theta}^{3}\right)[2]$ as

$$
\left\{A^{k} a^{\mu} b^{v} \mid k \geq 0, \mu, v \in \mathbb{Z}\right\} \cup\left\{B^{k} a^{\mu} b^{v} \mid k>0, \mu, v \in \mathbb{Z}\right\} .
$$

Here for $\mu, \nu<0$ we have written $b^{\mu}:=b^{*|\mu|}$ and $a^{\nu}:=a^{*|v|}$ for brevity. The $C^{*}$ algebra of the Heegaard quantum sphere $C\left(S_{p q \theta}^{3}\right)$ can also be defined as the universal $C^{*}$-algebra for the relations (0.16). One can set the parameters $p$ and $q$ equal to zero without changing this $C^{*}$-algebra, and prove that it is isomorphic with a certain pullback $C^{*}$-algebra [2].

Let $\mathcal{O}(U(1))$ be the coordinate $*$-Hopf algebra of $U(1)$ generated by a unitary $u$. The coaction of $\mathcal{O}(U(1))$ on $\mathcal{O}\left(S_{p q \theta}^{3}\right)$ is defined on generators by $\rho(a):=a \otimes u$, $\rho(b):=b \otimes u$. This coaction defines a $\mathbb{Z}$-grading $\operatorname{deg}: \mathcal{O}\left(S_{p q \theta}^{3}\right) \rightarrow \mathbb{Z}$ on $\mathcal{O}\left(S_{p q \theta}^{3}\right)$, with $\operatorname{deg}(a)=1=\operatorname{deg}(b)$. Note that all the basis elements in (0.18) have a definite grading degree. The coaction $\rho$ can be equivalently written as an action

$$
\alpha: U(1) \longrightarrow \operatorname{Aut}\left(\mathcal{O}\left(S_{p q \theta}^{3}\right)\right), \quad \alpha_{e^{i \varphi}}(a)=e^{i \varphi} a, \quad \alpha_{e^{i \varphi}}(b)=e^{i \varphi} b .
$$

This action extends to the $C^{*}$-algebra $C\left(S_{p q \theta}^{3}\right)$. One can prove that the algebra of coaction-invariant (or action-invariant) elements is generated as a $*$-algebra by $A, B$, and $z:=a b^{*}$. They satisfy the relations

$$
\begin{gathered}
A^{*}=A, \quad B^{*}=B, \quad A B=0, \quad A z=p z A, \quad z B=q B z, \\
z^{*} z=1-p A-B, \quad z z^{*}=1-A-q B .
\end{gathered}
$$

The universal $*$-algebra for these relations coincides with the coaction-invariant subalgebra. We call it the coordinate algebra of a mirror quantum sphere [13]. Note that by [9], $\mathcal{O}\left(S_{p q \theta}^{3}\right)$ is a piecewise trivial principal comodule algebra. The covering is given by a pair of ideals $\mathcal{O}\left(S_{p q \theta}^{3}\right) A$ and $\mathcal{O}\left(S_{p q \theta}^{3}\right) B$. The quotients $\mathcal{O}\left(S_{p q \theta}^{3}\right) / \mathcal{O}\left(S_{p q \theta}^{3}\right) A$ and $\mathcal{O}\left(S_{p q \theta}^{3}\right) / \mathcal{O}\left(S_{p q \theta}^{3}\right) B$ are both given by quantum solid tori [2].

0.3.3. Heegaard quantum lens spaces $[13,19]$. The $*$-Hopf algebra $\mathcal{O}(\mathbb{Z} / N \mathbb{Z})$ is generated by a unitary element $\tilde{u}$ satisfying $\tilde{u}^{N}=1$. There is a natural surjection $\pi: \mathcal{O}(U(1)) \rightarrow \mathcal{O}(\mathbb{Z} / N \mathbb{Z})$ given by $u \mapsto \tilde{u}$. This surjection defines a coaction of $\mathcal{O}(\mathbb{Z} / N \mathbb{Z})$ on $\mathcal{O}\left(S_{p q \theta}^{3}\right)$. The coaction-invariant subspace for this $\mathcal{O}(\mathbb{Z} / N \mathbb{Z})$ coaction is simply the subspace of elements of degree divisible by $N$. We denote this algebra by $\mathcal{O}\left(L_{p q \theta}^{N}\right)$, and call $L_{p q \theta}^{N}$ the Heegaard quantum lens space of type $N$. Likewise, we inject $\mathbb{Z} / N \mathbb{Z}$ into $U(1)$ via roots of unity, and use the action $\alpha$ to define the $\mathbb{Z} / N \mathbb{Z}$-invariant subalgebra of the $C^{*}$-algebra $C\left(S_{p q \theta}^{3}\right)$. We call the invariant subalgebra the $C^{*}$-algebra of the Heegaard quantum lens space of type $N$, and denote by $C\left(L_{p q \theta}^{N}\right)$. 
0.4. Odd-to-even connecting homomorphism. Consider a pullback diagram

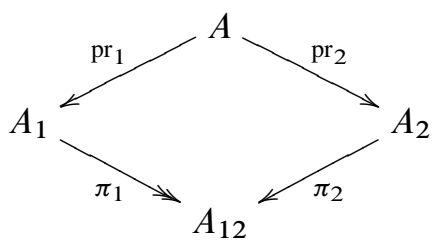

in the category of unital algebras. Explicitly, we can write

$$
A \cong\left\{\left(a_{1}, a_{2}\right) \in A_{1} \times A_{2} \mid \pi_{1}\left(a_{1}\right)=\pi_{2}\left(a_{2}\right)\right\}=\operatorname{Ker}\left(A_{1} \oplus A_{2} \stackrel{\left(\pi_{1},-\pi_{2}\right)}{\longrightarrow} A_{12}\right) .
$$

If one of the defining morphisms (here we choose $\pi_{1}$ ) is surjective, then there exists a long exact sequence in algebraic $K$-theory [16]

$$
\begin{aligned}
\cdots \longrightarrow K_{1}^{\mathrm{alg}}\left(A_{1} \oplus A_{2}\right) & \longrightarrow K^{\mathrm{alg}}\left(A_{12}\right) \\
& \stackrel{\partial_{10}^{\mathrm{alg}}}{\longrightarrow} K_{0}^{\mathrm{alg}}(A) \longrightarrow K_{0}^{\mathrm{alg}}\left(A_{1} \oplus A_{2}\right) \longrightarrow K_{0}^{\mathrm{alg}}\left(A_{12}\right) .
\end{aligned}
$$

The mapping $\partial_{10}^{\text {alg }}: K_{1}^{\text {alg }}\left(A_{12}\right) \longrightarrow K_{0}^{\text {alg }}(A)$ is obtained as follows. Take an invertible matrix $U \in G L_{n}\left(A_{12}\right)$ representing a class in $K_{1}^{\text {alg }}\left(A_{12}\right)$. There exist liftings $c, d \in$ $M_{n}\left(A_{1}\right)$ such that $\pi_{1}(c)=U^{-1}$ and $\pi_{1}(d)=U$. Then (e.g., see [6])

$$
p_{U}:=\left(\begin{array}{cc}
(c(2-d c) d, 1) & (c(2-d c)(1-d c), 0) \\
((1-d c) d, 0) & \left((1-d c)^{2}, 0\right)
\end{array}\right) \in M_{2 n}(A)
$$

is an idempotent matrix. The assignment

$$
\partial_{10}^{\mathrm{alg}}: K_{1}^{\mathrm{alg}}\left(A_{12}\right) \ni[U] \longmapsto\left[p_{U}\right]-\left[I_{n}\right] \in K_{0}^{\mathrm{alg}}(A),
$$

where $I_{n}$ is the identity matrix of the same size as the matrix $U$, gives an explicit form for the odd-to-even connecting homomorphism [16, Theorem 3.3, Page 28], which we fix for the remainder of the paper. We refer to this as the Milnor construction.

It is known that the odd-to-even connecting homomorphism exists also for the $K$-theory of $C^{*}$-algebras (cf. [7]), and is given by the same explicit formula. Since this formula is pivotal in proving our main result, for the sake of completeness, we provide its complete proof assuming it for the algebraic $K$-theory ${ }^{1}$. We proceed by translating the final part of the long exact sequence (0.22) into the $K$-theory of $C^{*}$ algebras. The $K_{0}$-groups are simply the same, and comparing the definitions of $K_{1}^{\text {alg }}$ and $K_{1}$ immediately yields a functorial surjection $K_{1}^{\text {alg }}(A) \ni[U] \mapsto[U] \in K_{1}(A)$ for any unital $C^{*}$-algebra $A$.

\footnotetext{
${ }^{1}$ This proof is a courtesy of Nigel Higson.
} 
Next, we want to split this surjection and define $K_{1}\left(A_{12}\right) \stackrel{\partial_{10}}{\longrightarrow} K_{0}(A)$ by composing such a set-theoretical splitting with $\partial_{10}^{\text {alg }}$. In order to show that it is independent of the choice of a splitting, we need to use the homotopy invariance of $K_{0}$. More precisely, if $\left[U_{0}\right]=\left[U_{1}\right] \in K_{1}\left(A_{12}\right)$, then there exists $n \in \mathbb{N}$ such that $\widetilde{U_{0}}:=\operatorname{diag}\left(U_{0}, I_{k}\right)$ and $\widetilde{U_{1}}:=\operatorname{diag}\left(U_{1}, I_{l}\right)$ are elements of $G L_{n}\left(A_{12}\right)$ that are homotopic via elements of $G L_{n}\left(A_{12}\right)$. In other words, there exists an invertible element $U$ in the $C^{*}$-algebra

$$
C\left([0,1], M_{n}\left(A_{12}\right)\right) \cong M_{n}\left(C\left([0,1], A_{12}\right)\right) \cong M_{n}\left(A_{12} \bar{\otimes} C([0,1])\right)
$$

satisfying $\operatorname{ev}_{0}(U)=\widetilde{U_{0}}$ and $\operatorname{ev}_{1}(U)=\widetilde{U_{1}}$, where $\operatorname{ev}_{t}$ stands for the evaluation map at $t$. Furthermore, since tensoring with nuclear $C^{*}$-algebras is always exact, we can conclude that $A \bar{\otimes} C([0,1])$ is isomorphic with the pullback

$$
\operatorname{Ker}\left(\left(A_{1} \bar{\otimes} C([0,1])\right) \oplus\left(A_{2} \bar{\otimes} C([0,1])\right) \stackrel{\left(\pi_{1},-\pi_{2}\right) \otimes \mathrm{id}}{\longrightarrow} A_{12} \bar{\otimes} C([0,1])\right) .
$$

This allows us to apply the Milnor construction to $U$ to obtain an idempotent $p_{U}$ in the $C^{*}$-algebra $M_{2 n}(A \bar{\otimes} C([0,1]))$. On the other hand, the evaluation maps ev , ev $_{1}$ : $A \bar{\otimes} C([0,1]) \rightarrow A$ are homotopic, so that, by the homotopy invariance of $K_{0}$, we conclude that

$$
\left[p_{\widetilde{U}_{0}}\right]=\operatorname{ev}_{0 *}\left[p_{U}\right]=\operatorname{ev}_{1 *}\left[p_{U}\right]=\left[p_{\widetilde{U_{1}}}\right] \in K_{0}(A)
$$

Consequently, we obtain

$$
\partial_{10}^{\mathrm{alg}}\left(\left[U_{0}\right]\right)=\partial_{10}^{\mathrm{alg}}\left(\left[\widetilde{U_{0}}\right]\right)=\left[p_{\widetilde{U_{0}}}\right]-\left[I_{n}\right]=\left[p_{\widetilde{U_{1}}}\right]-\left[I_{n}\right]=\partial_{10}^{\mathrm{alg}}\left(\left[\widetilde{U_{1}}\right]\right)=\partial_{10}^{\mathrm{alg}}\left(\left[U_{1}\right]\right)
$$

Thus we have defined a map

$$
\partial_{10}: K_{1}\left(A_{12}\right) \stackrel{\text { lift }}{\longrightarrow} K_{1}^{\mathrm{alg}}\left(A_{12}\right) \stackrel{\partial_{10}^{\mathrm{alg}}}{\longrightarrow} K_{0}^{\mathrm{alg}}(A)=K_{0}(A) .
$$

Since $\left[\operatorname{diag}\left(U, U^{\prime}\right)\right] \in K_{1}^{\mathrm{alg}}\left(A_{12}\right)$ is a lifting of $\left[\operatorname{diag}\left(U, U^{\prime}\right)\right] \in K_{1}\left(A_{12}\right)$, the map $\partial_{10}$ is automatically a group homomorphism. This leads to the following diagram:

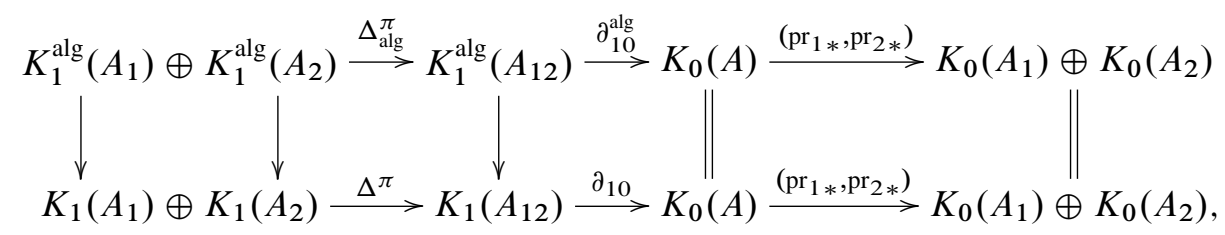

where we abbreviate $\Delta^{\pi}:=\pi_{1 *}-\pi_{2 *}$ and $\Delta_{\text {alg }}^{\pi}:=\pi_{1 * a l g}-\pi_{2 * a l g}$. Here the vertical arrows are canonical surjections. The commutativity of the first two squares follows from the functoriality of these surjections. The remaining two squares are commutative by construction. Now, the exactness of the top row (see (0.22)) and the 
surjectivity of all vertical arrows imply the exactness of the bottom row. Combining this with the Bott periodicity, we obtain the Mayer-Vietoris 6-term exact sequence $[18,1]$

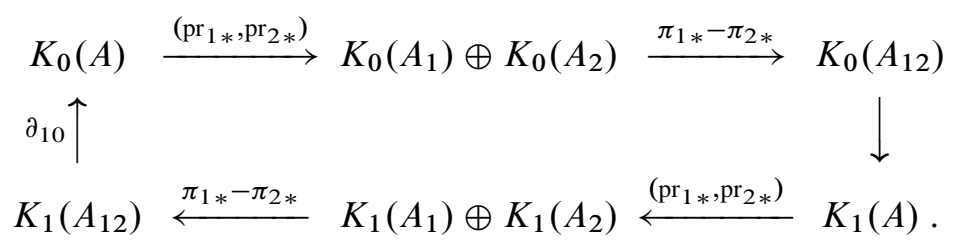

\section{Comodule algebras over the coordinate algebras of Heegaard lens spaces}

1.1. Polynomial identities for the quantum disc. Recall the definition of $p$-deformed binomial coefficients

$$
\left[\begin{array}{c}
n \\
m
\end{array}\right]_{p}:=\frac{[n]_{p} !}{[m]_{p} ![n-m]_{p} !},
$$

where $p$-deformed factorials $[n]_{p} !:=[1]_{p}[2]_{p} \ldots[n-1]_{p}[n]_{p}$ for $n-1 \in \mathbb{N}$, $[0]_{p} !:=1$, are defined in terms of $p$-deformed naturals $[n]_{p}:=1+p+p^{2}+$ $\ldots+p^{n-2}+p^{n-1}$ for $n-1 \in \mathbb{N},[0]_{p}:=0$.

Let $Y$ be a variable. Define a family of polynomials in $Y$, for $p \in \mathbb{R}_{+}$and $n-1 \in \mathbb{N}$, by the formulae

$$
\tilde{Q}_{n}^{p}(Y):=\sum_{m=1}^{n}(-1)^{m} p^{-n m+\frac{m(m+1)}{2}}\left[\begin{array}{l}
n \\
m
\end{array}\right]_{p} Y^{m} .
$$

Lemma 1.1. The polynomials (1.2) are uniquely determined by the recursive equations

$$
\tilde{Q}_{1}^{p}(Y)=-Y, \quad \tilde{Q}_{n+1}^{p}(Y)=(1-Y) \tilde{Q}_{n}^{p}\left(p^{-1} Y\right)-Y .
$$

Proof. We will proceed by induction. It follows from the definition of $\tilde{Q}_{n}^{p}$, Equation (1.2), that the case $n=1$ is satisfied. It is useful to rewrite the right-hand side of the second equation of (1.3) as follows:

$$
(1-Y) \tilde{Q}_{n}^{p}\left(p^{-1} Y\right)-Y=\tilde{Q}_{n}^{p}\left(p^{-1} Y\right)-Y \tilde{Q}_{n}^{p}\left(p^{-1} Y\right)-Y .
$$

For the first term we will separate the $m=1$ term from the sum defining $\tilde{Q}_{n}^{p}\left(p^{-1} Y\right)$, while for the second we will separate the $m=n$ term, and then renumber the sum. 
This yields

$$
\begin{aligned}
& (1-Y) \tilde{Q}_{n}^{p}\left(p^{-1} Y\right)-Y \\
& =(1-Y)\left(\sum_{m=1}^{n}(-1)^{m} p^{-n m+\frac{m(m+1)}{2}}\left[\begin{array}{l}
n \\
m
\end{array}\right]_{p} p^{-m} Y^{m}\right)-Y \\
& =-p^{-n}\left[\begin{array}{c}
n \\
1
\end{array}\right]_{p} Y-Y-(-1)^{n} p^{-\frac{n(n+1)}{2}}\left[\begin{array}{l}
n \\
n
\end{array}\right]_{p} Y^{n+1} \\
& +\sum_{m=2}^{n}\left((-1)^{m} p^{-n m+\frac{m(m+1)}{2}}\left[\begin{array}{l}
n \\
m
\end{array}\right]_{p} p^{-m}\right. \\
& \left.-(-1)^{m-1} p^{-n(m-1)+\frac{m(m-1)}{2}}\left[\begin{array}{c}
n \\
m-1
\end{array}\right]_{p} p^{-(m-1)}\right) Y^{m} \\
& =-p^{-n}\left(\left[\begin{array}{l}
n \\
1
\end{array}\right]_{p}+p^{n}\left[\begin{array}{l}
n \\
0
\end{array}\right]_{p}\right)-(-1)^{n} p^{-\frac{n(n+1)}{2}}\left[\begin{array}{l}
n+1 \\
n+1
\end{array}\right]_{p} Y^{n+1} \\
& +\sum_{m=2}^{n}(-1)^{m} p^{-(n+1) m+\frac{m(m+1)}{2}}\left(\left[\begin{array}{c}
n \\
m
\end{array}\right]_{p}+p^{n+1-m}\left[\begin{array}{c}
n \\
m-1
\end{array}\right]_{p}\right) Y^{m} \text {. }
\end{aligned}
$$

At this point we recall that for all $n \geq 0, m>0$, the deformed binomial coefficients satisfy the recursive formula

$$
\left[\begin{array}{l}
n \\
n
\end{array}\right]_{p}=\left[\begin{array}{l}
n \\
0
\end{array}\right]_{p}=1, \quad\left[\begin{array}{c}
n+1 \\
m
\end{array}\right]_{p}=\left[\begin{array}{l}
n \\
m
\end{array}\right]_{p}+p^{n+1-m}\left[\begin{array}{c}
n \\
m-1
\end{array}\right]_{p} .
$$

Applying this to our computation we obtain

$$
\begin{aligned}
& -p^{-n}\left[\begin{array}{c}
n+1 \\
1
\end{array}\right]_{p} Y+\sum_{m=2}^{n}(-1)^{m} p^{-(n+1) m+\frac{m(m+1)}{2}}\left[\begin{array}{c}
n+1 \\
m
\end{array}\right]_{p} Y^{m} \\
& \quad-(-1)^{n} p^{-\frac{n(n+1)}{2}}\left[\begin{array}{c}
n+1 \\
n+1
\end{array}\right]_{p} Y^{n+1} \\
& =\sum_{m=1}^{n+1}(-1)^{m} p^{-(n+1) m+\frac{m(m+1)}{2}}\left[\begin{array}{c}
n+1 \\
m
\end{array}\right]_{p} Y^{m}=Q_{n+1}^{p}(Y) .
\end{aligned}
$$

This completes the proof.

Lemma 1.2. For all $m, n \in \mathbb{N} \backslash\{0\}$, the family of polynomials $\left\{\tilde{Q}_{k}^{p}\right\}_{k}$ satisfies

$$
\tilde{Q}_{m+n}^{p}(Y)=\left(1+\tilde{Q}_{m}^{p}(Y)\right) \tilde{Q}_{n}^{p}\left(p^{-m} Y\right)+\tilde{Q}_{m}^{p}(Y) .
$$

Proof. We prove the above formula for arbitrary $n \in \mathbb{N}$ by induction on $m$. The case $m=1$ is true by Lemma 1.1. For the inductive step, suppose that Equation (1.8) is 
satisfied for some $m>0$. Then using Lemma 1.1 yields

$$
\begin{aligned}
(1 & \left.+\tilde{Q}_{m+1}^{p}(Y)\right) \tilde{Q}_{n}^{p}\left(p^{-(m+1)} Y\right)+\tilde{Q}_{m+1}^{p}(Y) \\
& =\left(1+(1-Y) \tilde{Q}_{m}^{p}\left(p^{-1} Y\right)-Y\right) \tilde{Q}_{n}^{p}\left(p^{-1-m} Y\right)+(1-Y) \tilde{Q}_{m}^{p}\left(p^{-1} Y\right)-Y \\
& =(1-Y)\left(\left(1+\tilde{Q}_{m}^{p}\left(p^{-1} Y\right)\right) \tilde{Q}_{n}^{p}\left(p^{-1-m} Y\right)+\tilde{Q}_{m}^{p}\left(p^{-1} Y\right)\right)-Y \\
& =(1-Y) \tilde{Q}_{n+m}^{p}\left(p^{-1} Y\right)-Y \\
& =\tilde{Q}_{n+m+1}^{p}(Y),
\end{aligned}
$$

as desired.

We now define polynomials for all $\mu \in \mathbb{Z}$ by the formulae

$$
Q_{\mu}^{p}(Y):= \begin{cases}\tilde{Q}_{\mu}^{p}(Y) & \text { if } \mu>0 \\ 0 & \text { if } \mu=0 \\ \tilde{Q}_{-\mu}^{p^{-1}}(p Y) & \text { if } \mu<0\end{cases}
$$

Note that the polynomials $Q_{-\mu}^{p}$ for $\mu>0$ satisfy the recursive relations

$$
Q_{-1}^{p}(Y)=-p Y, \quad Q_{-\mu-1}^{p}(Y)=(1-p Y) Q_{-\mu}^{p}(p Y)-p Y .
$$

Lemma 1.3. The generators $x$ and $x^{*}$ of the quantum disc $\mathcal{O}\left(D_{p}\right)$ satisfy the relations

$$
x^{\mu} x^{-\mu}=1+Q_{\mu}^{p}(X), \quad \mu \in \mathbb{Z}, \quad x^{-n}:=x^{* n}, \quad n \in \mathbb{N} .
$$

Proof. We proceed by induction on $|\mu|$, and begin by observing that the Formula (1.12) is immediately true for $\mu=0, \pm 1$. Suppose the formula is satisfied for some $\mu>0$. Then, using Lemma 1.1 yields

$$
\begin{aligned}
x^{\mu+1} x^{*(\mu+1)} & =x\left(x^{\mu} x^{* \mu}\right) x^{*} \\
& =x\left(1+Q_{\mu}^{p}(X)\right) x^{*} \\
& =x x^{*}\left(1+Q_{\mu}^{p}\left(p^{-1} X\right)\right) \\
& =(1-X)\left(1+Q_{\mu}^{p}\left(p^{-1} X\right)\right) \\
& =1+Q_{\mu+1}^{p}(X) .
\end{aligned}
$$

The proof for $\mu<0$ proceeds in the same way, because the identities for deformed factorials and binomial coefficients are the same for $0<p<1$ and $p>1$.

Let us define the family $Q_{\mu ; \nu}^{p}$ of polynomials, for all $\mu, v \in \mathbb{Z}$ by the formulae

$$
Q_{\mu ; \nu}^{p}(Y):= \begin{cases}0 & \text { if } \mu \nu \geq 0, \\ Q_{\mu}^{p}(Y) & \text { if } \mu \nu<0 \text { and }|\mu| \leq|v|, \\ Q_{-v}^{p}\left(p^{-(\mu+v)} Y\right) & \text { if } \mu \nu<0 \text { and }|\mu|>|v| .\end{cases}
$$

Now we can generalise Lemma 1.3. 
Lemma 1.4. Let $X:=1-x x^{*}, \mu, v \in \mathbb{Z}$, and for $\mu<0$ write $x^{\mu}:=x^{*|\mu|}$. Then the generators of the algebra $\mathcal{O}\left(D_{p}\right)$ satisfy

$$
x^{\mu} x^{\nu}=\left(1+Q_{\mu ; \nu}^{p}(X)\right) x^{\mu+v} .
$$

Proof. The statement is obvious if $\mu \nu \geq 0$, and we now consider the two cases when $\mu \nu<0$. First, for $|\mu| \leq|v|$ we find

$$
x^{\mu} x^{\nu}=\left(x^{\mu} x^{-\mu}\right) x^{\mu+\nu}=\left(1+Q_{\mu}^{p}(X)\right) x^{\mu+\nu}=\left(1+Q_{\mu ; \nu}^{p}(X)\right) x^{\mu+\nu} .
$$

Next, for $|\mu|>|\nu|$ we obtain

$$
\begin{aligned}
x^{\mu} x^{\nu} & =x^{\mu+v}\left(x^{-v} x^{\nu}\right)=x^{\nu+\mu}\left(1+Q_{-v}^{p}(X)\right) \\
& =\left(1+Q_{-v}^{p}\left(p^{-(v+\mu)} X\right)\right) x^{\nu+\mu}=\left(1+Q_{\mu ; \nu}^{p}(X)\right) x^{v+\mu},
\end{aligned}
$$

as needed.

1.2. Heegaard quantum lens spaces in terms of generators and relations. In what follows we will frequently need the following formula. Let $x$ and $y$ be two elements in an algebra such that $x y=e^{i \varphi} y x$, where $\varphi \in \mathbb{R}$. Then

$$
x^{\mu} y^{\mu}=e^{i \varphi \frac{\mu(\mu-1)}{2}}(x y)^{\mu},
$$

for $\mu \in \mathbb{Z}, x^{-n}:=\left(x^{*}\right)^{n}, y^{-n}:=\left(y^{*}\right)^{n}, n \in \mathbb{N}$.

We recall the coaction of $\mathcal{O}(\mathbb{Z} / N \mathbb{Z})$ on $\mathcal{O}\left(S_{p q \theta}^{3}\right)$ from Subsection 0.3.3. The coaction-invariant subspace of this coaction is simply the subspace of elements of degree divisible by $N$, and it is called a lens space $L_{p q \theta}^{N}$ of type $N$. It follows from $(0.18)$ that $\mathcal{O}\left(L_{p q \theta}^{N}\right)$ is spanned as a vector space by the set

$$
\begin{aligned}
\left\{A^{k} a^{\mu} b^{v} \mid k, \lambda, \mu, v\right. & \in \mathbb{Z}, k \geq 0, \mu+v=\lambda N\} \\
\cup & \left\{B^{k} a^{\mu} b^{v} \mid k, \lambda, \mu, v \in \mathbb{Z}, k>0, \mu+v=\lambda N\right\},
\end{aligned}
$$

where for $\mu, v<0$ we have written $b^{\mu}:=b^{*|\mu|}$ and $a^{\nu}:=a^{*|v|}$ for brevity. Let us also define

$$
\tilde{a}:=a^{N}, \quad \tilde{b}:=b^{N}, \quad z:=a b^{*} .
$$

It is not difficult to verify that the elements $A, B, z, \tilde{a}, \tilde{b}$ satisfy the commutation 
relations

$$
\begin{gathered}
A^{*}=A, \quad B^{*}=B, \quad A B=0, \quad A z=p z A, \quad z B=q B z, \\
z^{*} z=1-p A-B, \quad z z^{*}=1-A-q B, \\
A \tilde{a}=p^{N} \tilde{a} A, \quad A \tilde{b}=\tilde{b} A, \quad B \tilde{a}=\tilde{a} B, \quad B \tilde{b}=q^{N} \tilde{b} B, \\
z \tilde{a}=e^{i N 2 \pi \theta} \tilde{a} z, \quad z \tilde{b}^{*}=e^{-i N 2 \pi \theta} \tilde{b}^{*} z, \\
z \tilde{a}^{*}-e^{-i N 2 \pi \theta} \tilde{a}^{*} z=e^{-i N(N+1) \pi \theta}\left(p^{N}-1\right) A z^{1-N} \tilde{b}^{*}, \\
z b-e^{i N 2 \pi \theta} b z=e^{i \pi \theta N(N-1)} q\left(q^{-N}-1\right) B z^{1-N} \tilde{a}, \\
\tilde{a} \tilde{b}=e^{i N^{2} 2 \pi \theta} \tilde{b} \tilde{a}, \quad \tilde{a} \tilde{b}^{*}=e^{-i N^{2} 2 \pi \theta} \tilde{b}^{*} \tilde{a}, \quad \tilde{a} \tilde{b}^{*}=e^{-i \pi \theta N(N-1)} z^{N}, \\
\tilde{a}^{*} \tilde{a}=1+Q_{-N}^{p}(A), \quad \tilde{a} \tilde{a}^{*}=1+Q_{N}^{p}(A), \\
\tilde{b}^{*} \tilde{b}=1+Q_{-N}^{q}(B), \quad \tilde{b} \tilde{b}^{*}=1+Q_{N}^{q}(B) .
\end{gathered}
$$

Here the polynomials $Q_{\mu}^{p}$ were defined in Equation (1.10). Formulas (1.21a)(1.21d) and the first two equations in (1.21g) are straightforward consequences of Equations (0.16). In order to prove the last equality in (1.21g), we use (1.18). Equalities (1.21h)-(1.21i) follow immediately from Lemma 1.3. In order to prove Equation (1.21e), we need to do a little work. First we note that for all $n>0$ we have

$$
a a^{* n}-a^{* n} a=\left(p^{n}-1\right) A a^{*(n-1)} .
$$

Indeed, this formula holds for $n=1$, and for $n>1$ we can write

$$
\begin{aligned}
a a^{* n}-a^{* n} a & =\left(a a^{*}\right) a^{*(n-1)}-a^{*(n-1)}\left(a^{*} a\right) \\
& =(1-A) a^{*(n-1)}-a^{*(n-1)}(1-p A) \\
& =-A a^{*(n-1)}+p p^{n-1} A a^{*(n-1)} \\
& =\left(p^{n}-1\right) A a^{*(n-1)} .
\end{aligned}
$$

Now we are ready to prove Equation (1.21e). Using Equation (1.22) in step $(a)$ and Lemma 1.4 in step $(b)$, we compute

$$
\begin{aligned}
z \tilde{a}^{*}-e^{-i N 2 \pi \theta} \tilde{a}^{*} z & =a b^{-1} a^{-N}-e^{-i N 2 \pi \theta} a^{-N} a b^{-1} \\
& =e^{-i N 2 \pi \theta}\left(a a^{-N}-a^{-N} a\right) b^{-1} \\
& \stackrel{(a)}{=} e^{-i N 2 \pi \theta}\left(p^{N}-1\right) A a^{1-N} b^{-1} \\
& \stackrel{(b)}{=} e^{-i N 2 \pi \theta}\left(p^{N}-1\right) A a^{1-N}\left(b^{N-1} b^{-N}-Q_{N-1 ;-N}^{q}(B) b^{-1}\right) .
\end{aligned}
$$

Next, we use the fact that $A Q_{N-1 ;-N}^{q}(B)=0$ (due to $A B=0$ ) and commutation 
relations for $A$ to obtain

$$
\begin{aligned}
z \tilde{a}^{*}- & e^{-i N 2 \pi \theta} \tilde{a}^{*} z \\
& =e^{-i N 2 \pi \theta}\left(p^{N}-1\right) A\left(a^{1-N} b^{N-1}\right) b^{-N} \\
& =e^{-i N 2 \pi \theta-i(N-1)^{2} 2 \pi \theta}\left(p^{N}-1\right) A\left(a^{N-1} b^{-(N-1)}\right)^{*} b^{-N} \\
& =e^{-i N 2 \pi \theta-i(N-1)^{2} 2 \pi \theta+i \pi \theta(N-1)(N-2)}\left(p^{N}-1\right) A\left(\left(a b^{*}\right)^{N-1}\right)^{*} b^{-N} \\
& =e^{-i N(N+1) \pi \theta}\left(p^{N}-1\right) A z^{1-N} \tilde{b}^{*} .
\end{aligned}
$$

Here the second last equality follows from (1.18). The proof of Equation (1.21f) is similar.

We are now ready for the main claim of this subsection.

Theorem 1.5. Let $A$ be the universal $*$-algebra generated by the elements $\tilde{a}^{\prime}, \tilde{b}^{\prime}, z^{\prime}$, $A^{\prime}$ and $B^{\prime}$, and satisfying the same relations (1.21) as their unprimed counterparts. Then $\mathcal{O}\left(L_{p q \theta}^{N}\right)$ and $\mathcal{A}$ are isomorphic as $*$-algebras, and the set of vectors

$$
\mathscr{B}:=\left\{\left(A^{\prime}\right)^{k}\left(z^{\prime}\right)^{\mu}\left(\tilde{b}^{\prime}\right)^{v} \mid k>0, \mu, v \in \mathbb{Z}\right\} \cup\left\{\left(B^{\prime}\right)^{k}\left(z^{\prime}\right)^{\mu}\left(\tilde{a}^{\prime}\right)^{v} \mid k \geq 0, \mu, v \in \mathbb{Z}\right\}
$$

is a basis of AA. Here for $\mu, \nu<0$ we have written $\left(\tilde{b}^{\prime}\right)^{\mu}:=\left(\tilde{b}^{\prime}\right)^{*|\mu|},\left(\tilde{a}^{\prime}\right)^{\nu}:=\left(\tilde{a}^{\prime}\right)^{*|\nu|}$ and $\left(z^{\prime}\right)^{\mu}:=\left(z^{\prime}\right)^{*|\mu|}$ for brevity.

The proof of this theorem will occupy the remainder of this section. Until the final stage of the proof, we will abuse notation by dropping the primes on the generators of $\mathcal{A}$. First we will prove some additional commutation relations.

Lemma 1.6. Let $\mu, v \in \mathbb{Z}$, and for $\mu, v<0$ write $\tilde{b}^{\mu}:=\tilde{b}^{*|\mu|}$ and $\tilde{a}^{v}:=\tilde{a}^{*|v|}$. Then we have the relations

$$
\begin{gathered}
z^{\mu} z^{v}=\left(1+Q_{\mu ; \nu}^{p}(A)+Q_{-\mu ;-v}^{q}(B)\right) z^{\mu+v}, \\
\tilde{a}^{\mu} \tilde{a}^{v}=\left(1+Q_{N \mu ; N v}^{p}(A)\right) \tilde{a}^{\mu+v}, \\
\tilde{b}^{\mu} \tilde{b}^{v}=\left(1+Q_{N \mu ; N v}^{q}(B)\right) \tilde{b}^{\mu+v}, \\
A \tilde{a}^{v}=e^{-i \pi \theta N v(N \nu-1)} A z^{N v} \tilde{b}^{v}, \\
A z^{v} \tilde{b}^{\mu}=e^{i N 2 \pi \theta \mu v} A \tilde{b}^{\mu} z^{v},
\end{gathered}
$$

where the polynomials $Q_{\mu ; \nu}^{p}$ were defined in (1.14).

Proof. We prove each of the Equations (1.27) separately. For Equation (1.27a), we first prove, by induction, the simpler result

$$
z^{n} z^{* n}=1+Q_{n}^{p}(A)+Q_{-n}^{q}(B), \quad z^{* n} z^{n}=1+Q_{-n}^{p}(A)+Q_{n}^{p}(B) .
$$


Equations (1.28) is clearly satisfied for $n=0,1$ by Equations (1.21b) and (1.10). We will prove the first equality, the second being proved similarly. So suppose that $n>0$. Then

$$
\begin{aligned}
z^{n+1} z^{*(n+1)} & =z\left(z^{n} z^{* n}\right) z^{*} \\
& =z\left(1+Q_{n}^{p}(A)+Q_{-n}^{q}(B)\right) z^{*} \\
& =z z^{*}\left(1+Q_{n}^{p}\left(p^{-1} A\right)+Q_{-n}^{q}(q B)\right) \\
& =(1-A-q B)\left(1+Q_{n}^{p}\left(p^{-1} A\right)+Q_{-n}^{q}(q B)\right) \\
& =1+\left((1-A) Q_{n}^{p}\left(p^{-1} A\right)-A\right)+\left((1-q B) Q_{-n}^{q}(q B)-q B\right) \\
& =1+Q_{n+1}^{p}(A)+Q_{-(n+1)}^{q}(B),
\end{aligned}
$$

where in the last equality we used the recursive relations (1.3) and (1.11). Now Equation (1.27a) clearly holds when $\mu \nu \geq 0$. When $\mu \nu<0$ there are four cases, and we will show how the proof works in a single instance. Suppose then, that $|\mu| \leq|v|$ and $v<0$. Then using (1.28) and (1.14) we obtain

$$
\begin{aligned}
z^{|\mu|} z^{*|v|} & =\left(z^{|\mu|} z^{*|\mu|}\right) z^{*(|v|-|\mu|)} \\
& =\left(1+Q_{\mu}^{p}(A)+Q_{-\mu}^{q}(B)\right) z^{*(|v|-|\mu|)} \\
& =\left(1+Q_{\mu ; \nu}^{p}(A)+Q_{-\mu ;-v}^{q}(B)\right) z^{*(|v|-|\mu|)} .
\end{aligned}
$$

Equations $(1.27 \mathrm{~b})$ and $(1.27 \mathrm{c})$ are proved in the same way, and we just prove Equation (1.27b). First we prove the formulae

$$
\tilde{a}^{n} \tilde{a}^{* n}=1+Q_{N n}^{p}(A), \quad \tilde{a}^{* n} \tilde{a}^{n}=1+Q_{-N n}^{p}(A),
$$

by induction. By $(1.21 \mathrm{~h})$, these formulae are true for $n=0,1$. For $n>0$ we use Formula (1.8) and Equation (1.10) to find

$$
\begin{aligned}
\tilde{a}^{n+1} \tilde{a}^{*(n+1)} & =\tilde{a} \tilde{a}^{n} \tilde{a}^{* n} \tilde{a}^{*} \\
& =\tilde{a}\left(1+Q_{N n}^{p}(A)\right) \tilde{a}^{*} \\
& =\tilde{a} \tilde{a}^{*}\left(1+Q_{N n}^{p}\left(p^{-N} A\right)\right) \\
& =\left(1+Q_{N}^{p}(A)\right)\left(1+Q_{N n}^{p}\left(p^{-N} A\right)\right) \\
& =1+Q_{N(n+1)}^{p}(A) .
\end{aligned}
$$

Similarly

$$
\begin{aligned}
\tilde{a}^{*(n+1)} \tilde{a}^{n+1} & =\tilde{a}^{*}\left(1+Q_{-n N}^{p}(A)\right) \tilde{a} \\
& =\left(1+Q_{-N}^{p}(A)\right)\left(1+Q_{-n N}^{p}\left(p^{N} A\right)\right) \\
& =1+Q_{-N(n+1)}^{p}(A) .
\end{aligned}
$$

Now the full Formula (1.27b) can be easily proven using (1.31) and (1.14) by applying similar methods to those used to prove Equation (1.27a). 
To prove Equation (1.27d), first note that, as a direct consequence of (1.18) and (1.21g), for all $\mu, n \in \mathbb{Z}, n>0$, we obtain

$$
\tilde{a}^{\mu} \tilde{b}^{-\mu}=e^{-i \pi \theta N \mu(N \mu-1)} z^{N \mu}, \quad \tilde{a}^{-n}:=\tilde{a}^{* n}, \quad \tilde{b}^{-n}:=\tilde{b}^{* n} .
$$

Then Equation (1.27c) and Equation (1.34) yield

$$
\begin{aligned}
A \tilde{a}^{\mu} & =A \tilde{a}^{\mu} \tilde{b}^{-\mu} \tilde{b}^{\mu}-A \tilde{a}^{\mu} Q_{-\mu N ; \mu N}^{q}(B) \\
& =A\left(\tilde{a}^{\mu} \tilde{b}^{-\mu}\right) \tilde{b}^{\mu}-0 \\
& =e^{-i \pi \theta N \mu(N \mu-1)} A z^{N \mu} \tilde{b}^{\mu} .
\end{aligned}
$$

Finally, Equation (1.27e) follows directly from the commutation relations between $\tilde{b}^{ \pm 1}$ and $z^{ \pm 1}$ (Equations (1.21d), (1.21f)) and the fact that the additional term which might appear as a side effect of commuting $\tilde{b}^{ \pm 1}$ with $z^{ \pm 1}$ is proportional to $B$.

Let $\mathcal{V}$ be the linear subspace of $\mathcal{A}$ spanned by $\mathscr{B}$. Our aim is to show first that the generators of $\mathcal{A}$ belong to $\mathcal{V}$. Then, using some additional commutation relations, we will show that $\mathcal{V}$ is closed under multiplication, and hence equal to $\mathcal{A}$. Finally, we will argue that $\mathcal{A}$ is isomorphic to $\mathcal{O}\left(L_{p q \theta}^{N}\right)$. First we prove that $\tilde{b}, \tilde{b}^{*} \in \mathcal{V}$. Equations (1.21) allow us to write

$$
\tilde{b}=\tilde{b}\left(\tilde{a}^{*} \tilde{a}-Q_{-N}^{p}(A)\right)=e^{i \pi \theta N(N-1)} z^{* N} \tilde{a}-Q_{-N}^{p}(A) \tilde{b} .
$$

Similarly $\tilde{b}^{*}=\tilde{b}^{*}\left(\tilde{a} \tilde{a}^{*}-Q_{N}^{p}(A)\right)=e^{i \pi \theta N(N+1)} z^{N} \tilde{a}^{*}-Q_{N}^{p}(A) \tilde{b}^{*}$, which completes the argument, since $Q_{N}^{P}(A)$ has no constant term.

The previous argument, along with the definition of $\mathcal{V}$, shows that all the generators $\tilde{a}, \tilde{b}, z, A, B$, are contained in $\mathcal{V}$. Thus to show that $\mathcal{V}=\mathcal{A}$ we just need to prove that $\mathcal{V}$ is closed under multiplication. To this end, let us denote for brevity the following linear subspaces of $\mathcal{V}$ :

$$
\begin{aligned}
& \mathcal{V}_{A}:= \operatorname{Span}\left\{A^{k} z^{\mu} \tilde{b}^{v} \mid k, \mu, v \in \mathbb{Z}, k>0\right\}, \\
& \mathcal{V}_{0}:=\operatorname{Span}\left\{z^{\mu} \tilde{a}^{v} \mid \mu, v \in \mathbb{Z}\right\}, \\
& \mathcal{V}_{B}:= \operatorname{Span}\left\{B^{k} z^{\mu} \tilde{a}^{v} \mid k, \mu, v \in \mathbb{Z}, k>0\right\}, \\
& \mathcal{W}:=\operatorname{Span}\left(\mathcal{V}_{0} \cup \mathcal{V}_{B}\right) .
\end{aligned}
$$

Here for $\mu, v<0$ we have written $\tilde{b}^{\mu}:=\tilde{b}^{*|\mu|}, \tilde{a}^{v}:=\tilde{a}^{*|v|}$ and $z^{\mu}:=z^{*|\mu|}$ for brevity. The relation between these subspaces and $\mathcal{V}$ is

$$
\mathcal{V}=\operatorname{Span}\left(\mathcal{V}_{A} \cup \mathcal{W}\right)=\operatorname{Span}\left(\mathcal{V}_{A} \cup \mathcal{V}_{0} \cup \mathcal{V}_{B}\right) .
$$

Lemma 1.7. For all $v \in \mathbb{Z}$, we have the inclusions

$$
\begin{array}{ll}
\mathcal{V}_{A} \tilde{b}^{v} \subseteq \mathcal{V}_{A}, & \tilde{b}^{v} \mathcal{V}_{A} \subseteq \mathcal{V}_{A}, \\
\mathcal{V}_{A} z^{v} \subseteq \mathcal{V}_{A}, & z^{v} \mathcal{V}_{A} \subseteq \mathcal{V}_{A}, \\
\mathcal{V}_{A} \tilde{a}^{v} \subseteq \mathcal{V}_{A}, & \tilde{a}^{v} \mathcal{V}_{A} \subseteq \mathcal{V}_{A} .
\end{array}
$$


Proof. It is enough to consider an arbitrary vector from the set spanning $\mathcal{V}_{A}$, namely $A^{k} z^{\sigma} \tilde{b}^{\tau}$, where $k, \sigma, \tau \in \mathbb{Z}$ and $k>0$. It follows from Equation (1.27c) that $A^{k} z^{\sigma} \tilde{b}^{\tau} \tilde{b}^{v}=A^{k} z^{\sigma} \tilde{b}^{\tau+v} \in \mathcal{V}_{A}$. Similarly, using equations (1.27e) and (1.27c), we obtain

$$
\tilde{b}^{v} A^{k} z^{\sigma} \tilde{b}^{\tau}=A^{k} \tilde{b}^{v} z^{\sigma} \tilde{b}^{\tau}=e^{-i N 2 \pi \theta v \sigma} A^{k} z^{\sigma} \tilde{b}^{\nu} \tilde{b}^{\tau}=e^{-i N 2 \pi \theta v \sigma} A^{k} z^{\sigma} \tilde{b}^{\nu+\tau} \in \mathcal{V}_{A} .
$$

Next, using equations (1.27a) and (1.27e), we get

$$
A^{k} z^{\sigma} \tilde{b}^{\tau} z^{v}=e^{-i N 2 \pi \theta \tau v} A^{k} z^{\sigma} z^{v} \tilde{b}^{\tau}=e^{-i N 2 \pi \theta \tau v} A^{k}\left(1+Q_{\sigma ; v}^{p}(A)\right) z^{\sigma+v} \tilde{b}^{\tau} \in \mathcal{V}_{A} .
$$

Similarly, Equation (1.27a) yields

$$
z^{v} A^{k} z^{\sigma} \tilde{b}^{\tau}=p^{-v k} A^{k} z^{v} z^{\sigma} \tilde{b}^{\tau}=p^{-v k} A^{k}\left(1+Q_{\nu ; \sigma}^{p}(A)\right) z^{v+\sigma} \tilde{b}^{\tau} \in \mathcal{V}_{A} .
$$

The final inclusions follows immediately from Equations (1.39b) and (1.39a) using Equation (1.27d).

Lemma 1.8. For all $\mu, v \in \mathbb{Z}$, we have the commutation relation

$$
\tilde{a}^{v} z^{\mu}-e^{-i N 2 \pi \theta \mu v} z^{\mu} \tilde{a}^{v} \in \mathcal{V}_{A} .
$$

Here for $\mu, v<0$ we have written $\tilde{a}^{v}:=\tilde{a}^{*|v|}$ and $z^{\mu}:=z^{*|\mu|}$ for brevity.

Proof. The cases $\mu$ and $\nu$ both positive or both negative follow immediately from Equation (1.21d), even replacing $\mathcal{V}_{A}$ by the zero subspace. For $\mu>0$ and $v<0$, the result follows from Equation (1.21e). The final case follows by taking the adjoint of Equation (1.21e), manipulating the result using Equations (1.21a), (1.21d), and then finally applying Equation (1.27e).

Lemma 1.9. The vector subspace $\mathcal{V} \subseteq \mathcal{A}$ is closed under multiplication.

Proof. It is enough to consider products of basis vectors. First we note that because the only cost of commuting $A$ and $B$ through any other generator of $\mathcal{V}$ is the appearing of central coefficients and $A B=B A=0$, we can easily conclude that $\mathcal{V}_{A} \mathcal{V}_{B}=$ $\mathcal{V}_{B} \mathcal{V}_{A}=0$. Next, from Lemma 1.7 one immediately concludes that $\mathcal{V}_{A} \cdot \mathcal{V}_{A} \subseteq \mathcal{V}_{A}$, $\mathcal{V}_{A} \cdot \mathcal{V}_{0} \subseteq \mathcal{V}_{A}$ and $\mathcal{V}_{0} \cdot \mathcal{V}_{A} \subseteq \mathcal{V}_{A}$.

Furthermore, using (1.41), (1.39), (1.27a), (1.27b), one can infer that, for all $\mu, \nu, \sigma, \tau \in \mathbb{Z}$,

$$
\begin{aligned}
& z^{\mu} \tilde{a}^{v} z^{\sigma} \tilde{a}^{\tau} \\
& \quad \in e^{-i N 2 \pi \theta v \sigma} z^{\mu} z^{\sigma} \tilde{a}^{v} \tilde{a}^{\tau}+z^{\mu} \mathcal{V}_{A} \tilde{a}^{\tau} \\
& \quad \subseteq e^{-i N 2 \pi \theta v \sigma} z^{\mu} z^{\sigma} \tilde{a}^{v} \tilde{a}^{\tau}+\mathcal{V}_{A} \\
& \quad=e^{-i N 2 \pi \theta v \sigma}\left(1+Q_{\mu ; \sigma}^{p}(A)+Q_{-\mu ;-\sigma}^{q}(B)\right) z^{\mu+\sigma}\left(1+Q_{N \nu ; N \tau}^{p}(A)\right) \tilde{a}^{v+\tau}+\mathcal{V}_{A} \\
& \quad=e^{-i N 2 \pi \theta v \sigma}\left(1+Q_{\mu, \sigma}^{v, \tau}(A)+Q_{-\mu ;-\sigma}^{q}(B)\right) z^{\mu+\sigma} \tilde{a}^{v+\tau}+\mathcal{V}_{A},
\end{aligned}
$$


where we have denoted $Q_{\mu, \sigma}^{\nu, \tau}(A):=Q_{\mu ; \sigma}^{p}(A)+Q_{N \nu ; N \tau}^{p}\left(p^{-\mu-\sigma} A\right)\left(1+Q_{\mu ; \sigma}^{p}(A)\right)$ for brevity. Observe that due to Equation (1.27d) we have $Q_{\mu, \sigma}^{v, \tau}(A) z^{\mu+\sigma} \tilde{a}^{\nu+\tau} \in \mathcal{V}_{A}$, so that $z^{\mu} \tilde{a}^{\nu} z^{\sigma} \tilde{a}^{\tau} \in \mathcal{V}$ for all $\mu, \nu, \sigma, \tau \in \mathbb{Z}$. It follows immediately that also $\mathcal{W} \cdot \mathcal{W} \subseteq \mathcal{V}$, which completes the proof.

Summarising, we conclude that $\mathcal{V}=\mathcal{A}$ because $\mathcal{V}$ contains generators of $\mathcal{A}$ and is closed under multiplication. Hence the vectors in the set $\mathscr{B}$, Equation (1.26), span $\mathcal{A}$. This proves half of Theorem 1.5, and we now complete the proof. To this end, we take the natural $*$-homomorphism $f: \mathcal{A} \rightarrow \mathcal{O}\left(L_{p q \theta}^{N}\right)$ defined on generators of $\mathscr{A}$ by

$A^{\prime} \mapsto A:=1-a a^{*}, \quad B^{\prime} \mapsto B:=1-b b^{*}, \quad z^{\prime} \mapsto a b^{*}, \quad \tilde{a}^{\prime} \mapsto a^{N}, \quad \tilde{b}^{\prime} \mapsto b^{N}$.

It is enough to prove that this $*$-homomorphism is a linear bijection.

Before doing so, we note that it follows from (0.16) and (1.18) that

$$
f\left(\left(z^{\prime}\right)^{\mu}\right)=e^{i \pi \theta \mu(\mu-1)} a^{\mu} b^{-\mu}, \quad \mu \in \mathbb{Z} .
$$

Also, it follows from Equation (1.15) and relations (0.16), that the values of $f$ on linear generators (1.26) of $\mathcal{A}$ are given by

$$
\begin{gathered}
f\left(\left(A^{\prime}\right)^{k}\left(z^{\prime}\right)^{\mu}\left(\tilde{b}^{\prime}\right)^{v}\right)=e^{i \pi \theta \mu(\mu-1)} A^{k} a^{\mu} b^{N \nu-\mu}, \\
f\left(\left(B^{\prime}\right)^{k}\left(z^{\prime}\right)^{\mu}\left(\tilde{a}^{\prime}\right)^{\nu}\right)=e^{i 2 \pi \theta\left(\frac{\mu(\mu-1)}{2}+N \mu \nu\right)} B^{k} a^{\mu+N v} b^{-\mu}, \\
f\left(\left(z^{\prime}\right)^{\mu}\left(\tilde{a}^{\prime}\right)^{v}\right)=e^{i 2 \pi \theta\left(\frac{\mu(\mu-1)}{2}+N \mu \nu\right)}\left(1+Q_{\mu ; N v}^{p}(A)\right) a^{\mu+N v} b^{-\mu},
\end{gathered}
$$

for all $k, \mu, v \in \mathbb{Z}, k>0$.

First we show that the homomorphism $f$ is surjective. It is enough to prove that an arbitrary vector from basis (1.19) is in the image of $f$. For all $k, \mu, v \in \mathbb{Z}, k>0$, we have

$$
A^{k} a^{\mu} b^{N \nu-\mu}=f\left(e^{-i \pi \theta \mu(\mu-1)}\left(A^{\prime}\right)^{k}\left(z^{\prime}\right)^{\mu}\left(\tilde{b}^{\prime}\right)^{\nu}\right) .
$$

On the other hand, (1.44b) implies that for all $k, \mu, v \in \mathbb{Z}, k>0$, we have

$$
\begin{aligned}
& B^{k} a^{\mu} b^{N \nu-\mu} \\
& =f\left(e^{-i 2 \pi \theta\left(\frac{(-N v+\mu)(-N v+\mu-1)}{2}+N(-N v+\mu) v\right)}\left(B^{\prime}\right)^{k}\left(z^{\prime}\right)^{-N v+\mu}\left(\tilde{a}^{\prime}\right)^{v}\right) .
\end{aligned}
$$

Finally, it follows from the same computation which led to Equation (1.46) that for all $\mu, v \in \mathbb{Z}$

$$
\begin{array}{r}
f\left(e^{-i 2 \pi \theta\left(\frac{(-N v+\mu)(-N v+\mu-1)}{2}+N(-N v+\mu) v\right)}\left(z^{\prime}\right)^{-N \nu+\mu}\left(\tilde{a}^{\prime}\right)^{v}\right) \\
=\left(1+Q_{-N \nu+\mu ; N v}^{p}(A)\right) a^{\mu} b^{N \nu-\mu} .
\end{array}
$$


Hence, using Equation (1.45) we find that for all $\mu, v \in \mathbb{Z}$

$$
\begin{aligned}
a^{\mu} b^{N \nu-\mu}= & f\left(e^{-i 2 \pi \theta\left(\frac{(-N v+\mu)(-N v+\mu-1)}{2}+N(-N v+\mu) v\right)}\left(z^{\prime}\right)^{-N v+\mu}\left(\tilde{a}^{\prime}\right)^{v}\right) \\
& -f\left(e^{-i \pi \theta \mu(\mu-1)} Q_{-N v+\mu ; N v}^{p}\left(A^{\prime}\right)\left(z^{\prime}\right)^{\mu}\left(\tilde{b}^{\prime}\right)^{\nu}\right) .
\end{aligned}
$$

Next, to show that the homomorphism $f$ is injective, we suppose that $f(v)=0$ for some $v \in \mathcal{A}$. Since the set $\mathscr{B}$ spans $\mathcal{A}$, we can write $v$ as a linear combination

$$
\begin{aligned}
v & =\sum_{k>0 ; \mu, v \in \mathbb{Z}} \alpha_{k \mu \nu}\left(A^{\prime}\right)^{k}\left(z^{\prime}\right)^{\mu}\left(\tilde{b}^{\prime}\right)^{v}+\sum_{k^{\prime}>0 ; \mu^{\prime}, v^{\prime} \in \mathbb{Z}} \beta_{k^{\prime} \mu^{\prime} v^{\prime}}\left(B^{\prime}\right)^{k^{\prime}}\left(z^{\prime}\right)^{\mu^{\prime}}\left(\tilde{a}^{\prime}\right)^{v^{\prime}} \\
& +\sum_{\mu^{\prime \prime}, v^{\prime \prime} \in \mathbb{Z}} \gamma_{\mu^{\prime \prime} v^{\prime \prime}}\left(z^{\prime}\right)^{\mu^{\prime \prime}}\left(\tilde{a}^{\prime}\right)^{v^{\prime \prime}} .
\end{aligned}
$$

Using equations (1.44), we can explicitly compute $f(v)$ to be

$$
\begin{aligned}
& f\left(\sum_{k>0 ; \mu, v \in \mathbb{Z}} \alpha_{k \mu v}\left(A^{\prime}\right)^{k}\left(z^{\prime}\right)^{\mu}\left(\tilde{b}^{\prime}\right)^{v}\right. \\
& +\sum_{k^{\prime}>0 ; \mu^{\prime}, v^{\prime} \in \mathbb{Z}} \beta_{k^{\prime} \mu^{\prime} v^{\prime}}\left(B^{\prime}\right)^{k^{\prime}}\left(z^{\prime}\right)^{\mu^{\prime}}\left(\tilde{a}^{\prime}\right)^{v^{\prime}} \\
& \left.\quad+\sum_{\mu^{\prime \prime}, v^{\prime \prime} \in \mathbb{Z}} \gamma_{\mu^{\prime \prime} v^{\prime \prime}}\left(z^{\prime}\right)^{\mu^{\prime \prime}}\left(\tilde{a}^{\prime}\right)^{v^{\prime \prime}}\right) \\
& =\sum_{k>0 ; \mu, v \in \mathbb{Z}} \alpha_{k \mu \nu} e^{i \pi \theta \mu(\mu-1)} A^{k} a^{\mu} b^{N v-\mu} \\
& +\sum_{k^{\prime}>0 ; \mu^{\prime}, v^{\prime} \in \mathbb{Z}} \beta_{k^{\prime} \mu^{\prime} v^{\prime}} e^{i 2 \pi \theta\left(\frac{\mu^{\prime}\left(\mu^{\prime}-1\right)}{2}-N \mu^{\prime} v^{\prime}\right)} B^{k^{\prime}} a^{\mu^{\prime}+N v^{\prime}} b^{-\mu^{\prime}} \\
& \quad+\sum_{\mu^{\prime \prime}, v^{\prime \prime} \in \mathbb{Z}} \gamma_{\mu^{\prime \prime} v^{\prime \prime}} e^{i 2 \pi \theta\left(\frac{\mu^{\prime \prime}\left(\mu^{\prime \prime}-1\right)}{2}-N \mu^{\prime \prime} v^{\prime \prime}\right)}\left(1+Q_{\mu^{\prime \prime} ; N v^{\prime \prime}}^{p}(A)\right) a^{\mu^{\prime \prime}+N v^{\prime \prime}} b^{-\mu^{\prime \prime}}
\end{aligned}
$$

Since the set of vectors (1.19) is linearly independent, it follows immediately that $f(v)=0$ implies that $\beta_{k^{\prime} \mu^{\prime} v^{\prime}}=0$, for all $k^{\prime}, \mu^{\prime}, v^{\prime} \in \mathbb{Z}, k^{\prime}>0$. Now considering the terms $\gamma_{\mu^{\prime \prime} v^{\prime \prime}} a^{\mu^{\prime \prime}+N v^{\prime \prime}} b^{-\mu^{\prime \prime}}$ in the last sum, we see that $\gamma_{\mu^{\prime \prime} v^{\prime \prime}}=0$, for all $\mu^{\prime \prime}, v^{\prime \prime} \in \mathbb{Z}$. Then also $\alpha_{k \mu \nu}=0$, for all $k, \mu, v \in \mathbb{Z}, k>0$. Hence $v=0$, so that $f$ is injective. Finally, note that this also proves that the set of vectors (1.26) is linearly independent.

1.3. Non-cleftness of the Heegaard $\mathcal{O} \mathbb{Z} / N \mathbb{Z})$-comodule algebras. Let us begin by showing that $\mathcal{O}\left(S_{p q}^{3}\right)$ is a principal comodule algebra. As explained in the preliminaries, to this end it suffices to construct a strong connection. It turns out that a 
simple modification of the formulae for a strong connection given in [11, (4.4)-(4.6)] yields a strong connection in our case. We define the linear map

$$
\ell: \mathcal{O}(\mathbb{Z} / N \mathbb{Z}) \ni h \longmapsto \ell(h)=: h^{\langle 1\rangle} \otimes h^{\langle 2\rangle} \in \mathcal{O}\left(S_{p q \theta}^{3}\right) \otimes \mathcal{O}\left(S_{p q \theta}^{3}\right)
$$

(summation understood in $h^{\langle 1\rangle} \otimes h^{\langle 2\rangle}$ ) by setting its values on the basis elements $\tilde{u}^{n}$, $n=0, \ldots, N-1$, to be

$$
\ell(1):=1 \otimes 1, \ell(\tilde{u}):=a^{*} \otimes a+p^{-1} b^{*} A \otimes b, \ell\left(\tilde{u}^{n}\right):=\tilde{u}^{\langle 1\rangle} \ell\left(\tilde{u}^{n-1}\right) \tilde{u}^{\langle 2\rangle},
$$

$n=2, \ldots, N-1$. Proving that the above defined map is a strong connection is almost identical to an argument provided in [11].

The method we use to show the non-existence of a cleaving map, is to prove that there are not enough invertibles in the comodule algebra of the quantum sphere to accomodate the range of such a map.

Theorem 1.10. The only invertible elements in the algebra of polynomial functions on the Heegaard quantum sphere $\mathcal{O}\left(S_{p q \theta}^{3}\right)$ are non-zero multiples of the identity.

Proof. Our proof will follow the general idea and structure of the proof of nonexistence of non-trivial invertible elements in another noncommutative deformation of the polynomial algebra of $S^{3}$, namely the Hopf algebra $\mathcal{O}\left(S L_{q}(2)\right)$ [10]. Here we use the basis (0.18) to present each element in $\mathcal{O}\left(S_{p q \theta}^{3}\right)$ as a linear combination of monomials $a^{\mu} b^{v}, \mu, v \in \mathbb{Z}$, with coefficients in the polynomial algebra generated by $A$ and $B$. The crux of the proof is that $a$ and $b$ are invertible up to polynomials in $A$ and $B$.

For the duration of this proof, for $\mathbb{Z} \ni \mu, \nu<0$ we will write $b^{\mu}:=b^{*|\mu|}$ and $a^{\nu}:=a^{*|v|}$ for brevity. Recall that any element $r \in \mathcal{O}\left(S_{p q \theta}^{3}\right)$ can be expanded using basis (0.18) as

$$
r=\sum_{\substack{\mu, v \in \mathbb{Z} \\ \text { finite }}} C_{\mu ; \nu}^{r}(A, B) a^{\mu} b^{\nu},
$$

where $\left\{C_{\mu ; \nu}^{r}\right\}_{\mu, \nu \in \mathbb{Z}}$ is a family of complex polynomials in two variables without mixed monomials. Here no mixed monomials means that it can be written as

$$
C_{\mu ; \nu}^{r}(X, Y)=\gamma_{\mu ; \nu}^{r}+\alpha_{\mu ; \nu}^{r}(X)+\beta_{\mu ; \nu}^{r}(Y),
$$

where $\gamma_{\mu ; \nu}^{r} \in \mathbb{C}$ and $\alpha_{\mu ; \nu}^{r}$ and $\beta_{\mu ; \nu}^{r}$ are polynomials such that $\alpha_{\mu ; \nu}^{r}(0)=0$ and $\beta_{\mu ; \nu}^{r}(0)=0$. Since the family of vectors $(0.18)$ is a linear basis, it follows that the polynomials $C_{\mu ; \nu}^{r}$ are uniquely determined by $r$.

We endow $\mathbb{Z} \times \mathbb{Z}$ with the lexicographical order, i.e.,

$$
(\mu, v) \leq\left(\mu^{\prime}, v^{\prime}\right):=\quad \mu<\mu^{\prime} \vee\left(\mu=\mu^{\prime} \wedge v \leq v^{\prime}\right) .
$$

This order is linear (total) and satisfies

$$
(\mu, v) \leq\left(\mu^{\prime}, v^{\prime}\right) \Longleftrightarrow\left(-\mu^{\prime},-v^{\prime}\right) \leq(-\mu,-v) .
$$


The latter justifies introducing a notation $-(\mu, \nu)=(-\mu,-v)$. Next, we define a $\mathbb{Z}^{2}$-grading deg : $\mathcal{O}\left(S_{p q \theta}^{3}\right) \rightarrow \mathbb{Z}^{2}$ by declaring

$$
\operatorname{deg}\left(C(A, B) a^{\mu} b^{v}\right):=(\mu, v), \quad \text { for all } \mu, v \in \mathbb{Z} \text { and } C(A, B) \neq 0,
$$

where $C$ is a polynomial in two variables with coefficients in $\mathbb{C}$.

We will also need to divide $\mathcal{O}\left(S_{p q \theta}^{3}\right)$ into the following linear subspaces:

$$
\begin{aligned}
\mathcal{X} & :=\operatorname{Span}\left\{A^{k} a^{\mu} b^{v} \mid k \geq 0, \mu, v \in \mathbb{Z}\right\}, \\
X_{1} & :=\operatorname{Span}\left\{A^{k} a^{\mu} b^{v} \mid k>0, \mu, v \in \mathbb{Z}\right\}, \\
y & :=\operatorname{Span}\left\{B^{k} a^{\mu} b^{v} \mid k \geq 0, \mu, v \in \mathbb{Z}\right\}, \\
y_{1} & :=\operatorname{Span}\left\{B^{k} a^{\mu} b^{v} \mid k>0, \mu, v \in \mathbb{Z}\right\} .
\end{aligned}
$$

Note that

$$
\mathcal{X} \cap y_{1}=\mathcal{X}_{1} \cap y=\{0\}, \quad x \oplus y_{1}=\mathcal{X}_{1} \oplus y=\mathcal{O}\left(S_{p q \theta}^{3}\right),
$$

i.e., we have two splittings of $\mathcal{O}\left(S_{p q \theta}^{3}\right)$ into a direct sum of subspaces. The expansion (1.53) and Formula (1.54) provide a way to split any element $r \in \mathcal{O}\left(S_{p q \theta}^{3}\right)$ into a sum of vectors from these subspaces. For instance, we can split $r$ into vectors

$$
\sum_{\mu, v \in \mathbb{Z}}\left(\gamma_{\mu ; \nu}^{r}+\alpha_{\mu ; \nu}^{r}(A)\right) a^{\mu} b^{\nu} \in \mathcal{X} \quad \text { and } \quad \sum_{\mu, \nu \in \mathbb{Z}} \beta_{\mu ; \nu}^{r}(B) a^{\mu} b^{\nu} \in y_{1} .
$$

Using commutation relations (0.16) and Equation (1.15), we can write the expansion of a product of elements $r$ and $s$ of $\mathcal{O}\left(S_{p q \theta}^{3}\right)$ as

$$
\begin{aligned}
& r s=\left(\sum_{\mu, v \in \mathbb{Z}} C_{\mu ; v}^{r}(A, B) a^{\mu} b^{v}\right)\left(\sum_{\mu^{\prime}, v^{\prime} \in \mathbb{Z}} C_{\mu^{\prime} ; v^{\prime}}^{s}(A, B) a^{\mu^{\prime}} b^{v^{\prime}}\right) \\
&= \sum_{\mu, v, \mu^{\prime}, v^{\prime} \in \mathbb{Z}} e^{i 2 \pi \theta v \mu^{\prime}} C_{\mu ; v}^{r}(A, B) C_{\mu^{\prime} ; v^{\prime}}^{s}\left(p^{-\mu} A, q^{-v} B\right)\left(a^{\mu} a^{\mu^{\prime}}\right)\left(b^{v} b^{v^{\prime}}\right) \\
&= \sum_{\mu, v, \mu^{\prime}, v^{\prime} \in \mathbb{Z}} e^{i 2 \pi \theta v \mu^{\prime}} C_{\mu ; v}^{r}(A, B) C_{\mu^{\prime} ; v^{\prime}}^{s}\left(p^{-\mu} A, q^{-v} B\right) \\
&\left(1+Q_{\mu ; \mu^{\prime}}^{p}(A)\right)\left(1+Q_{v ; v^{\prime}}^{q}(B)\right) a^{\mu+\mu^{\prime}} b^{v+v^{\prime}} .
\end{aligned}
$$

Hence we obtain

$$
\begin{gathered}
C_{\mu ; \nu}^{r s}(A, B)=\sum_{\mu^{\prime}, v^{\prime} \in \mathbb{Z}} e^{i 2 \pi \theta v^{\prime}\left(\mu-\mu^{\prime}\right)} C_{\mu^{\prime} ; v^{\prime}}^{r}(A, B) C_{\mu-\mu^{\prime} ; \nu-v^{\prime}}^{s}\left(p^{-\mu^{\prime}} A, q^{-v^{\prime}} B\right) \\
\left(1+Q_{\mu^{\prime} ; \mu-\mu^{\prime}}^{p}(A)+Q_{\nu^{\prime} ; \nu-v^{\prime}}^{q}(B)\right) .
\end{gathered}
$$


It follows immediately that

$$
x y_{1} \subseteq y_{1}, \quad y_{1} x \subseteq y_{1}, \quad y x_{1} \subseteq x_{1}, \quad x_{1} y \subseteq x_{1}
$$

Furthermore, writing

$$
r=\sum_{\substack{\mu, v \in \mathbb{Z} \\ \text { finite }}} C_{\mu ; \nu}^{r}(A, B) a^{\mu} b^{\nu}=\sum_{\substack{\mu, v \in \mathbb{Z} \\ \text { finite }}}\left(\gamma_{\mu ; \nu}^{r}+\alpha_{\mu ; \nu}^{r}(A)+\beta_{\mu ; \nu}^{r}(B)\right) a^{\mu} b^{v}
$$

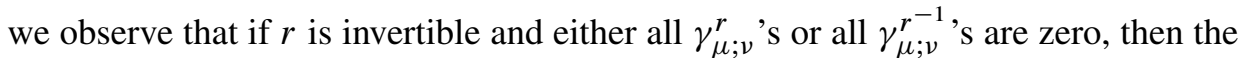
expansion of $r r^{-1}$ in terms of basis (0.18) would contain only vectors from $X_{1}$ or $\mathcal{Y}_{1}$. However, this is impossible because $1 \notin X_{1} \oplus \mathcal{Y}_{1}$. Hence, if $r$ is invertible, then at least one of the $\gamma_{\mu ; \nu}^{r}$ 's (and also one of the $\gamma_{\mu ; \nu}^{r^{-1}}$ 's) is non-zero. This observation allows us to define maps on the invertible elements of $\mathcal{O}\left(S_{p q \theta}^{3}\right)$ by

$$
\begin{aligned}
\max _{\operatorname{deg}}: \mathcal{O}\left(S_{p q \theta}^{3}\right) & \longrightarrow \mathbb{Z} \times \mathbb{Z}, \\
r & \longmapsto \max \left\{(\mu, v) \in \mathbb{Z} \times \mathbb{Z} \mid \gamma_{\mu ; \nu}^{r}+\alpha_{\mu ; \nu}^{r}(A) \neq 0\right\}, \\
\max \operatorname{deg}_{y}: \mathcal{O}\left(S_{p q \theta}^{3}\right) & \longrightarrow \mathbb{Z} \times \mathbb{Z}, \\
r & \longmapsto \max \left\{(\mu, v) \in \mathbb{Z} \times \mathbb{Z} \mid \gamma_{\mu ; \nu}^{r}+\beta_{\mu ; \nu}^{r}(B) \neq 0\right\}, \\
\min \operatorname{deg}_{x}: \mathcal{O}\left(S_{p q \theta}^{3}\right) & \longrightarrow \mathbb{Z} \times \mathbb{Z}, \\
r & \longmapsto \min \left\{(\mu, v) \in \mathbb{Z} \times \mathbb{Z} \mid \gamma_{\mu ; \nu}^{r}+\alpha_{\mu ; \nu}^{r}(A) \neq 0\right\}, \\
\min \operatorname{deg}_{y}: \mathcal{O}\left(S_{p q \theta}^{3}\right) & \longrightarrow \mathbb{Z} \times \mathbb{Z}, \\
r & \longmapsto \min \left\{(\mu, v) \in \mathbb{Z} \times \mathbb{Z} \mid \gamma_{\mu ; \nu}^{r}+\beta_{\mu ; \nu}^{r}(B) \neq 0\right\} .
\end{aligned}
$$

Next, let $r, s \in \mathcal{O}\left(S_{p q \theta}^{3}\right)$ be invertible, and let $(\mu, v)=\max _{\operatorname{deg}}(r)$ and $\left(\mu^{\prime}, v^{\prime}\right)=\max \operatorname{deg}_{X}(s)$. Then, by Equation (1.62),

$$
\begin{aligned}
& \gamma_{\mu+\mu^{\prime} ; \nu+v^{\prime}}^{r s}+\alpha_{\mu+\mu^{\prime} ; \nu+v^{\prime}}^{r s}(A) \\
& \quad=e^{i 2 \pi \theta v \mu^{\prime}}\left(\gamma_{\mu ; \nu}^{r}+\alpha_{\mu ; \nu}^{r}(A)\right)\left(\gamma_{\mu^{\prime} ; \nu^{\prime}}^{s}+\alpha_{\mu^{\prime} v^{\prime}}^{s}\left(p^{-\mu} A\right)\right)\left(1+Q_{\mu ; \mu^{\prime}}^{p}(A)\right) .
\end{aligned}
$$

The factors on the right-hand side are non-zero by the definition of $(\mu, v)$ and $\left(\mu^{\prime}, v^{\prime}\right)$ and because the algebra generated by $A$ does not contain zero-divisors. It follows that $\gamma_{\mu+\mu^{\prime} ; v+v^{\prime}}^{r s}+\alpha_{\mu+\mu^{\prime} ; v+v^{\prime}}^{r s}(A) \neq 0$. Therefore, for all invertible $r$ and $s$ we have

$$
\max \operatorname{deg}_{\mathcal{X}}(r s)=\max _{\operatorname{deg}}(r)+\max \operatorname{deg}_{\mathcal{X}}(s),
$$

where the addition of pairs of integers is done componentwise. Similarly, we prove that for all invertible $r$ and $s$ we have

$$
\begin{aligned}
\max \operatorname{deg}_{y}(r s) & =\max \operatorname{deg}_{y}(r)+\max \operatorname{deg}_{y}(s), \\
\min \operatorname{deg}_{X}(r s) & =\min \operatorname{deg}_{X}(r)+\min \operatorname{deg}_{x}(s), \\
\min \operatorname{deg}_{y}(r s) & =\min \operatorname{deg}_{y}(r)+\min _{\operatorname{deg}}(s) .
\end{aligned}
$$


Suppose now that $r \in \mathcal{O}\left(S_{p q \theta}^{3}\right)$ is invertible. Then

$$
\max \operatorname{deg}_{X}\left(r r^{-1}\right)=\max \operatorname{deg}_{X}(1)=(0,0),
$$

and similarly

$$
\max \operatorname{deg}_{y}\left(r r^{-1}\right)=\min \operatorname{deg}_{y}\left(r r^{-1}\right)=\min \operatorname{deg}_{\mathcal{X}}\left(r r^{-1}\right)=(0,0) .
$$

Hence the equations (1.67) imply that

$$
\begin{array}{rlrl}
\max \operatorname{deg}_{X}\left(r^{-1}\right) & =-\max _{\operatorname{deg}}(r), & \max \operatorname{deg}_{y}\left(r^{-1}\right) & =-\max \operatorname{deg}_{y}(r), \\
\min \operatorname{deg}_{X}\left(r^{-1}\right) & =-\min \operatorname{deg}_{X}(r), \quad \min \operatorname{deg}_{y}\left(r^{-1}\right)=-\min _{\operatorname{deg}_{y}(r) .}
\end{array}
$$

In particular, starting with an obvious property that

$$
\min \operatorname{deg}_{X}\left(r^{-1}\right) \leq \max \operatorname{deg}_{X}\left(r^{-1}\right)
$$

and substituting Equation (1.70) into it yields that

$$
-\min \operatorname{deg}_{X}(r) \leq-\max \operatorname{deg}_{X}(r) .
$$

Hence, using Equation (1.56), we obtain that $\max _{\operatorname{deg}}(r) \leq \min \operatorname{deg}_{X}(r)$, so that

$$
\max \operatorname{deg}_{\mathcal{X}}(r)=\min \operatorname{deg}_{\mathcal{X}}(r)=-\max \operatorname{deg}_{\mathcal{X}}\left(r^{-1}\right)=-\min \operatorname{deg}_{\mathcal{X}}\left(r^{-1}\right) .
$$

Similarly we prove that

$$
\max \operatorname{deg}_{y}(r)=\min \operatorname{deg}_{y}(r)=-\max \operatorname{deg}_{y}\left(r^{-1}\right)=-\min \operatorname{deg}_{y}\left(r^{-1}\right) .
$$

On the other hand, we already know that in the sum (1.64) $\exists(\mu, \nu): \gamma_{\mu ; \nu}^{r} \neq 0$. By the linear independence, also $\gamma_{\mu ; \nu}^{r}+\alpha_{\mu ; \nu}^{r}(A) \neq 0$ and $\gamma_{\mu ; \nu}^{r}+\beta_{\mu ; \nu}^{r}(B) \neq 0$. Therefore, by (1.73a) and (1.73b), max $\operatorname{deg}_{X}(r)=(\mu, \nu)=\max \operatorname{deg}_{y}(r)$. Using again the linear independence, we conclude that all terms in (1.64) with the index different from $(\mu, v)$ must vanish.

Summarising, so far we have proven that an invertible element $r \in \mathcal{O}\left(S_{p q \theta}^{3}\right)$ and its inverse must have the form

$$
\begin{aligned}
r & =\left(\gamma_{\mu ; \nu}^{r}+\alpha_{\mu ; \nu}^{r}(A)+\beta_{\mu ; \nu}^{r}(B)\right) a^{\mu} b^{\nu}, \\
r^{-1} & =\left(\gamma_{-\mu ;-\nu}^{r^{-1}}+\alpha_{-\mu ;-\nu}^{r^{-1}}(A)+\beta_{-\mu ;-\nu}^{r^{-1}}(B)\right) a^{-\mu} b^{-v}
\end{aligned}
$$

for some $\mu, v \in \mathbb{Z}$, with $\gamma_{\mu ; \nu}^{r} \gamma_{-\mu ;-\nu}^{r^{-1}} \neq 0$. Then inserting $r$ and $r^{-1}$ into Formula (1.62) yields

$$
\begin{array}{r}
1=e^{-i 2 \pi \theta \mu \nu}\left(\gamma_{\mu ; \nu}^{r}+\alpha_{\mu ; \nu}^{r}(A)\right)\left(\gamma_{-\mu ;-\nu}^{r^{-1}}+\alpha_{-\mu ;-\nu}^{r^{-1}}(A)\right)\left(1+Q_{\mu ;-\mu}^{p}(A)\right) \\
+B(\operatorname{polynomial}(B)) .
\end{array}
$$


By linear independence, the term in $B$ vanishes. Now by polynomial degree counting, we can conclude that the polynomial in $A$ is of degree zero, and hence so are its factors. This yields

$$
\alpha_{\mu ; \nu}^{r}(A)=\alpha_{-\mu ;-\nu}^{r^{-1}}(A)=Q_{\mu ;-\mu}^{p}(A)=0 .
$$

Repeating the argument for $B$ gives

$$
\beta_{\mu ; \nu}^{r}(B)=\beta_{-\mu ;-v}^{r^{-1}}(B)=Q_{\nu ;-\nu}^{q}(B)=0 .
$$

Recalling that $Q_{\mu ;-\mu}^{p}(A)=Q_{v ;-v}^{q}(B)=0$ only if $v=\mu=0$, we infer that $r=\gamma_{0 ; 0}^{r} \in \mathbb{C} \backslash\{0\}$.

Since the Hopf algebra $\mathcal{O}(\mathbb{Z} / N \mathbb{Z})$ contains a non-trivial group-like element, in the image of a cleaving map there would have to be a non-trivial invertible (see preliminaries). Hence Theorem 1.10 implies that a cleaving map does not exist:

Corollary 1.11. $\mathcal{O}\left(S_{p q \theta}^{3}\right)$ is a non-cleft $\mathcal{O}(\mathbb{Z} / N \mathbb{Z})$-comodule algebra.

\section{Comodule algebras over the $C^{*}$-algebras of Heegaard lens spaces}

2.1. K-groups. For the $K$-theory calculations to come, we utilise a description of the Heegaard quantum sphere as a pullback of $U(1)-C^{*}$-algebras, see the first example of Section 5.2 in [9]. We write $\mathcal{T}$ for the Toeplitz algebra, and since we will have two copies of this algebra, we denote their generating isometries by $z_{ \pm}$. The corresponding unitaries implementing the crossed products $\mathcal{T} \rtimes_{ \pm \theta} \mathbb{Z}$ are denoted by $u_{ \pm}$. Finally, $Z_{+}$and $U_{+}$stand for the two generating unitaries of the noncommutative torus $C\left(S^{1}\right) \rtimes_{\theta} \mathbb{Z}$. With this notation, the pullback structure of

$$
C\left(S_{p q \theta}^{3}\right) \cong\left\{\left(a_{+}, a_{-}\right) \in \mathcal{T} \rtimes_{\theta} \mathbb{Z} \oplus \mathcal{T} \rtimes_{-\theta} \mathbb{Z}: \pi_{1}\left(a_{+}\right)=\pi_{2}\left(a_{-}\right)\right\}
$$

is given by the following diagram and maps:

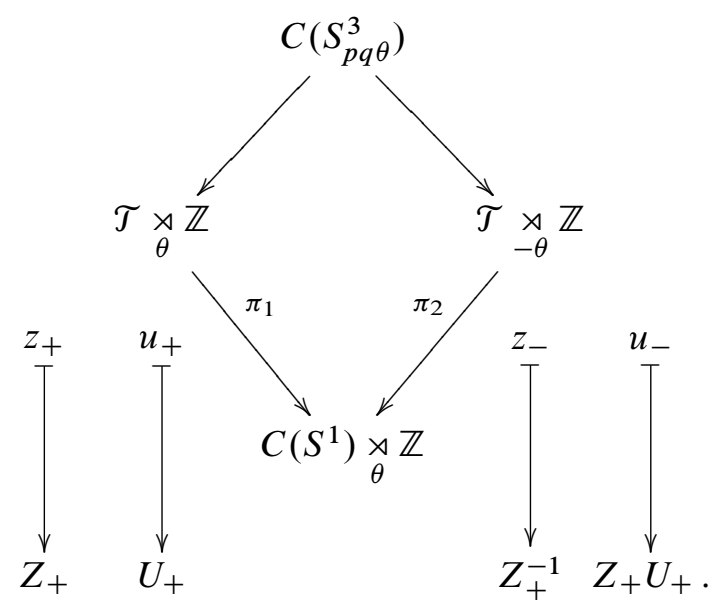


This is a pullback diagram of $U(1)-C^{*}$-algebras, with the natural $U(1)$-action on the $\mathbb{Z}$-parts. We restrict this action of $U(1)$ to $\mathbb{Z} / N \mathbb{Z}$ and consider the pullback diagram obtained by the restriction of the above one to its $\mathbb{Z} / N \mathbb{Z}$-invariant part:

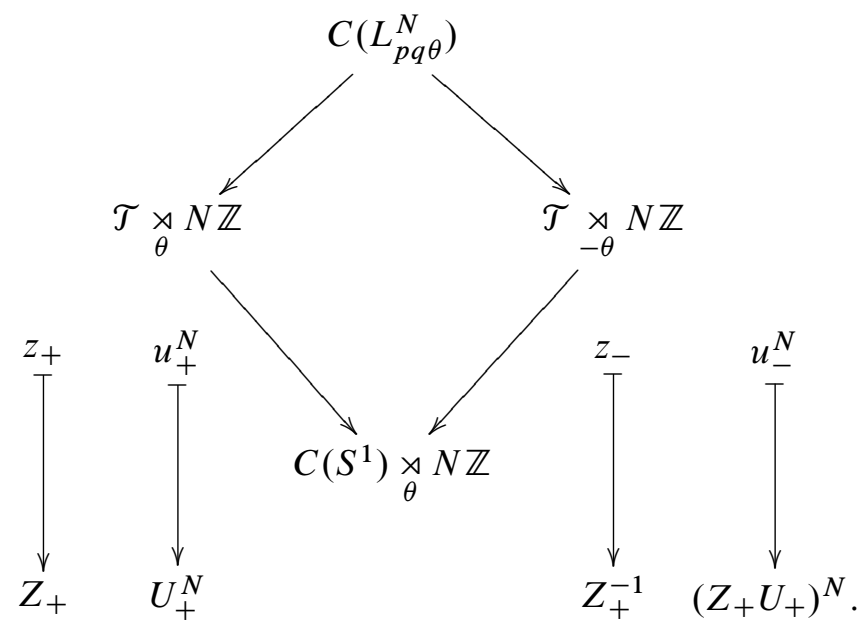

We can use the commutation relations in the noncommutative torus to simplify the rightmost map as $\left(Z_{+} U_{+}\right)^{N}=e^{i N(N-1) \pi \theta} Z_{+}^{N} U_{+}^{N}$. Introducing the generators

$$
\tilde{z}_{+}:=z_{+}, \quad \tilde{u}_{+}:=u_{+}^{N}, \quad \tilde{z}_{-}:=z_{-}, \quad \tilde{u}_{-}:=u_{-}^{N}, \quad Z:=Z_{+}, \quad U:=U_{+}^{N},
$$

we can rewrite this pullback diagram as

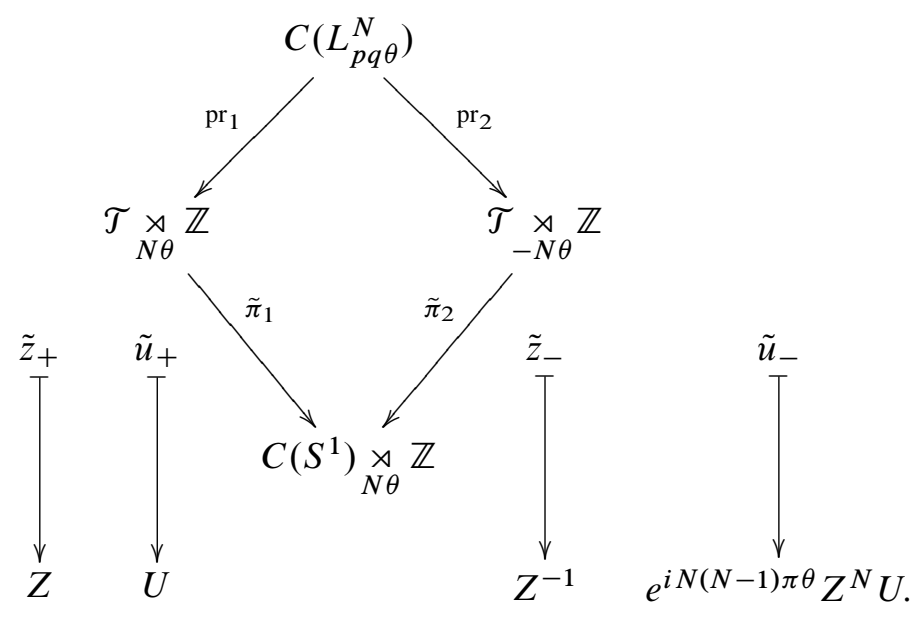

For the $K$-theory calculations to come, we need to know the effect of the maps in 
the pullback diagram on $K$-theory generators. These are given by

$$
\begin{aligned}
& K_{0}(\mathcal{T} \underset{N \theta}{\rtimes} \mathbb{Z}) \cong \mathbb{Z} \ni m \stackrel{\tilde{\pi}_{1 *}}{\longmapsto}(m, 0) \in \mathbb{Z} \oplus \mathbb{Z} \cong K_{0}\left(C\left(S^{1}\right) \underset{N \theta}{\rtimes} \mathbb{Z}\right), \\
& K_{0}(\underset{\mathcal{T}}{\rtimes} \underset{-N \theta}{\rtimes} \mathbb{Z}) \cong \mathbb{Z} \ni m \stackrel{\tilde{r}_{2 *}}{\longmapsto}(m, 0) \in \mathbb{Z} \oplus \mathbb{Z} \cong K_{0}\left(C\left(S^{1}\right) \underset{N \theta}{\rtimes} \mathbb{Z}\right), \\
& K_{1}(\mathcal{T} \underset{N \theta}{\rtimes} \mathbb{Z}) \cong \mathbb{Z} \ni n \stackrel{\tilde{\pi}_{1 *}}{\longmapsto}(0, n) \in \mathbb{Z} \oplus \mathbb{Z} \cong K_{1}\left(C\left(S^{1}\right) \underset{N \theta}{\rtimes} \mathbb{Z}\right), \\
& K_{1}(\underset{-N \theta}{\rtimes} \mathbb{Z}) \cong \mathbb{Z} \ni n \stackrel{\tilde{\pi}_{2 *}}{\longmapsto}(N n, n) \in \mathbb{Z} \oplus \mathbb{Z} \cong K_{1}\left(C\left(S^{1}\right) \underset{N \theta}{\rtimes} \mathbb{Z}\right) .
\end{aligned}
$$

Inserting these $K$-theory groups and maps into the Mayer-Vietoris 6-term exact sequence (see preliminaries)

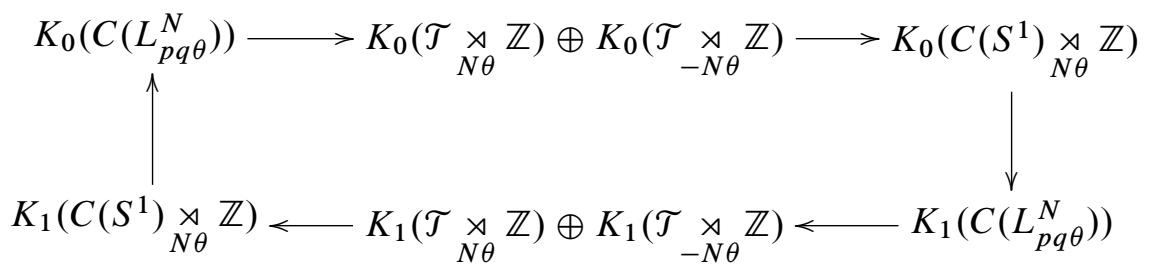

yields the exact sequence

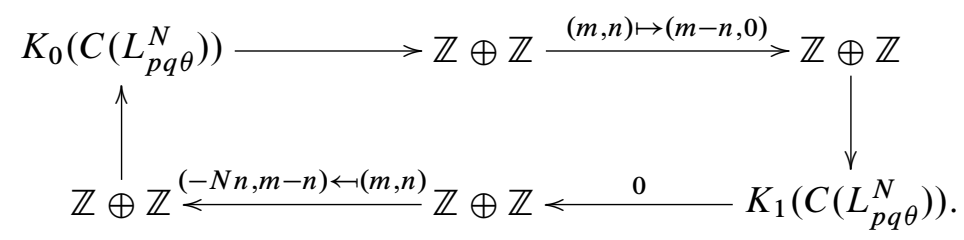

Thus we immediately obtain that $K_{1}\left(C\left(L_{p q \theta}^{N}\right)\right)=\mathbb{Z}$. Using this information, we can simplify the sequence (2.8) to the exact sequence

$$
0 \rightarrow N \mathbb{Z} \oplus \mathbb{Z} \hookrightarrow \mathbb{Z} \oplus \mathbb{Z} \rightarrow K_{0}\left(C\left(L_{p q \theta}^{N}\right)\right) \rightarrow \mathbb{Z} \oplus \mathbb{Z} \rightarrow \mathbb{Z} \rightarrow 0 .
$$

Consequently, the sequence

$$
0 \rightarrow N \mathbb{Z} \hookrightarrow \mathbb{Z} \stackrel{f}{\rightarrow} K_{0}\left(C\left(L_{p q \theta}^{N}\right)\right) \rightarrow \mathbb{Z} \rightarrow 0
$$

is exact. Since $\mathbb{Z}$ is projective, $K_{0}\left(C\left(L_{p q \theta}^{N}\right)\right)=\operatorname{Im} f \oplus \mathbb{Z}$ and $\operatorname{Im} f=\mathbb{Z} / N \mathbb{Z}$. Summarising, we have derived

Theorem 2.1. $K_{0}\left(C\left(L_{p q \theta}^{N}\right)\right)=\mathbb{Z} / N \mathbb{Z} \oplus \mathbb{Z}$ and $K_{1}\left(C\left(L_{p q \theta}^{N}\right)\right)=\mathbb{Z}$. 
2.2. The generators of $K_{\mathbf{0}}$. With the foregoing computation of $K$-groups at hand, we are ready to prove the main result of this paper.

Theorem 2.2. Let $L_{N}:=\left\{x \in C\left(S_{p q \theta}^{3}\right) \mid \alpha_{e^{\frac{2 \pi i}{N}}}(x)=e^{\frac{2 \pi i}{N}} x\right\} \subseteq C\left(S_{p q \theta}^{3}\right)$. Then $L_{N}$ is not stably free as a left $C\left(L_{p q \theta}^{N}\right)$-module.

Proof. The $C^{*}$-algebra $C\left(S_{p q \theta}^{3}\right)$ is isomorphic as a $U(1)-C^{*}$-algebra to $C\left(S_{00 \theta}^{3}\right)$ [2, Theorem 2.8]. The latter is generated by isometries $s$ and $t$ with the $U(1)$ action given by $\tilde{\alpha}_{e^{i \varphi}}(s)=e^{i \varphi} s, \tilde{\alpha}_{e^{i \varphi}}(t)=e^{i \varphi} t$. The induced $\mathbb{Z} / N \mathbb{Z}$-action can be therefore written as $\Delta_{R}(s)=s \otimes \tilde{u}, \Delta_{R}(t)=t \otimes \tilde{u}$, where $\tilde{u} \in C(\mathbb{Z} / N \mathbb{Z})$, $\tilde{u}\left(e^{\frac{2 \pi i k}{N}}\right)=e^{\frac{2 \pi i k}{N}}$. One can immediately check that the formula

$$
\ell\left(\tilde{u}^{k}\right):=s^{* k} \otimes s^{k}, \quad k \in\{0, \ldots, N-1\},
$$

defines a strong connection, so that $C\left(S_{p q \theta}^{3}\right)$ is a $C(\mathbb{Z} / N \mathbb{Z})$-principal comodule algebra (see preliminaries).

Next, let $\mathbb{C}$ be a left $C(\mathbb{Z} / N \mathbb{Z})$-comodule via $\varrho(1):=\tilde{u} \otimes 1$. Then, as explained in Subsection 0.2, we can write

$$
L_{N} \cong C\left(S_{p q \theta}^{3}\right)_{C(\mathbb{Z} / N \mathbb{Z})}^{\square} \mathbb{C} \cong C\left(L_{p q \theta}^{N}\right) s s^{*} .
$$

Thus $s s^{*} \in C\left(L_{p q \theta}^{3}(N)\right)$ is an idempotent (in fact, projection) representing the $K_{0^{-}}$ class of $L_{N}$. On the other hand, we know from the preceding $K$-theory computation that the $\mathbb{Z} / N \mathbb{Z}$-part of $K_{0}\left(C\left(L_{p q \theta}^{N}\right)\right)$ is generated by the odd-to-even connecting homomorphism applied to the $K_{1}$-class of the unitary $Z \in C\left(S^{1}\right) \rtimes_{N \theta} \mathbb{Z}$. In other words, $\partial_{10}[Z] \neq 0$ and $N \partial_{10}[Z]=0$. To compute $\partial_{10}[Z]$, we lift $Z$ and $Z^{-1}$ to $\tilde{z}_{+}, \tilde{z}_{+}^{*} \in \mathcal{T} \rtimes_{N \theta} \mathbb{Z}$ respectively. The Milnor construction (0.23) yields

$$
\left(\begin{array}{cc}
(1,1) & (0,0) \\
(0,0) & \left(1-\tilde{z}_{+} \tilde{z}_{+}^{*}, 0\right)
\end{array}\right)=\left(\begin{array}{cc}
1 & 0 \\
0 & 1
\end{array}\right)-\left(\begin{array}{cc}
0 & 0 \\
0 & \left(\tilde{z}_{+} \tilde{z}_{+}^{*}, 1\right)
\end{array}\right) .
$$

Finally, using the pullback description of $C\left(S_{00 \theta}^{3}\right)$ in Equation (2.1), we note that $s$ is expressed as $\left(z_{+} u_{+}, u_{-}\right)$. Hence $s s^{*}$ can be written as $\left(z_{+} z_{+}^{*}, 1\right)$. As this element belongs to the $\mathbb{Z} / N \mathbb{Z}$-invariant part, we can rewrite it in terms of the $C\left(L_{p q \theta}^{N}\right)=$ $C\left(S_{p q \theta}^{3}\right) \mathbb{Z} / N \mathbb{Z}$-generators, which in this instance just means adding $\tilde{\text {, so that }} s s^{*}=$ $\left(\tilde{z}_{+} \tilde{z}_{+}^{*}, 1\right)$. Now it is clear that

$$
\partial_{10}[Z]=2[1]-\left[L_{N}\right]-[1]=[1]-\left[L_{N}\right] .
$$

If $L_{N}$ were stably free, then there would exist $k, m \in \mathbb{N}$ such that $L_{N} \oplus C\left(L_{p q \theta}^{N}\right)^{k}$ $\cong C\left(L_{p q \theta}^{N}\right)^{m}$ as modules. Then the foregoing equation would imply

$$
\partial_{10}[Z]=[1]+k[1]-\left[L_{N} \oplus C\left(L_{p q \theta}^{N}\right)^{k}\right]=(k+1-m)[1] .
$$


However, since $\partial_{10}[Z] \neq 0$, we conclude that $k+1-m \neq 0$. Hence $N(k+1-m) \neq 0$ and $N(k+1-m)[1]=0$. This contradicts the fact that the projection map $C\left(L_{p q \theta}^{N}\right) \stackrel{\mathrm{pr}_{1}}{\longrightarrow} \mathcal{T} \rtimes_{N \theta} \mathbb{Z}$ takes the identity to the identity inducing the map

$$
\begin{gathered}
K_{0}\left(C\left(L_{p q \theta}^{N}\right)\right) \stackrel{\mathrm{pr}_{1 *}}{\longrightarrow} K_{0}(\mathcal{T} \underset{N \theta}{\rtimes} \mathbb{Z})=\mathbb{Z}[1], \\
N(k+1-m)[1] \stackrel{\mathrm{pr}_{1 *}}{\longmapsto} N(k+1-m)[1] \neq 0 .
\end{gathered}
$$

Hence $L_{N}$ is not stably free.

The above theorem shows that the module $L_{N}$ associated to the $C(\mathbb{Z} / N \mathbb{Z})$ principal comodule algebra $\left.C\left(S_{p q \theta}\right)\right)$ is responsible for the torsion part of the group $K_{0}\left(C\left(L_{p q \theta}^{N}\right)\right)$ and is not stably free. There is a hierarchy of implications: associated module not stably free $\Rightarrow$ associated module not free $\Rightarrow$ principal comodule algebra not cleft $\Rightarrow$ principal comodule algebra not trivial. In the algebraic part of this paper, we managed to prove by elementary methods that the $\mathcal{O}(\mathbb{Z} / N \mathbb{Z})$-principal comodule algebra $\mathcal{O}\left(S_{p q \theta}^{3}\right)$ is not cleft. Not going beyond algebraic methods, we could also prove that the associated $\mathcal{O}\left(L_{p q \theta}^{N}\right)$-module

$$
\mathscr{L}_{N}:=\left\{x \in \mathcal{O}\left(S_{p q \theta}^{3}\right) \mid \alpha_{e^{\frac{2 \pi i}{N}}}(x)=e^{\frac{2 \pi i}{N}} x\right\} \cong \mathcal{O}\left(S_{p q \theta}^{3}\right)_{C(\mathbb{Z} / N \mathbb{Z})}^{\square} \mathbb{C}
$$

is not free. However, to show that $\mathscr{L}_{N}$ is not stably free, we need to take advantage of Theorem 2.2.

Corollary 2.3. The $\mathcal{O}\left(L_{p q \theta}^{N}\right)$-module $\mathscr{L}_{N}$ is not stably free.

Proof. Replacing Formula (2.11) by (1.52) defines a strong connection on $C(\mathbb{Z} / N \mathbb{Z})$ principal comodule algebra $C\left(S_{p q \theta}^{3}\right)$. As in the proof of Theorem 2.2, we can use this strong connection to compute an idempotent matrix representing the associated $C\left(L_{p q \theta}^{N}\right)$-module $L_{N}$. It turns out to be

$$
e_{N}:=\left(\begin{array}{cc}
1-A & p^{-1} z A \\
z^{*} & p^{-1} A
\end{array}\right) \in M_{2}\left(\mathcal{O}\left(L_{p q \theta}^{N}\right)\right) \subseteq M_{2}\left(C\left(L_{p q \theta}^{N}\right)\right) .
$$

It follows from Theorem 2.2 that, for any non-negative integers $k$ and $l$, there are no matrices $v$ and $w$ over $C\left(L_{p q \theta}^{N}\right)$ such that

$$
v w=\left(\begin{array}{cc}
e_{N} & 0 \\
0 & I_{k}
\end{array}\right) \quad \text { and } \quad w v=I_{l},
$$

where $I_{k}$ and $I_{l}$ are identity matrices of the size $k$ and $l$ respectively. Hence there are no such matrices over $\mathcal{O}\left(L_{p q \theta}^{N}\right)$. On the other hand, since $\mathscr{L}_{N}$ is associated by the same group-like $\tilde{u}$ as $L_{N}$, and the same formulae (1.52) define a strong connection 
on both $\mathcal{O}\left(S_{p q \theta}^{3}\right)$ and $C\left(S_{p q \theta}^{3}\right)$ comodule algebras over $\mathcal{O}(\mathbb{Z} / N \mathbb{Z})=C(\mathbb{Z} / N \mathbb{Z})$, we infer that the idempotent matrix $e_{N}$ also represents $\mathscr{L}_{N}$ as an $\mathcal{O}\left(L_{p q \theta}^{N}\right)$-module. Combining these two facts, we conclude that the $\mathcal{O}\left(L_{p q \theta}^{N}\right)$-module $\mathscr{L}_{N}$ is not stably isomorphic to $\mathcal{O}\left(L_{p q \theta}^{N}\right)$ for any positive integer $l$.

\section{3. $U(1)$-prolongations}

3.1. Prolongations in the algebraic setting. By Subsection 0.2 , the prolongation of $\mathcal{O}\left(S_{p q \theta}^{3}\right)$ by $\mathcal{O}(U(1))$ is a principal comodule algebra. Furthermore, using [4, Proposition 4.1], one can prove that it is not cleft if there are no invertible elements in $\mathcal{O}\left(S_{p q \theta}^{3}\right)$ other then multiples of identity. Therefore, Theorem 1.10 enjoys the following corollary.

Corollary 3.1. The prolongation $\mathcal{O}\left(S_{p q \theta}^{3}\right) \underset{\mathcal{O}(\mathbb{Z} / N \mathbb{Z})}{\square} \mathcal{O}(\mathbb{Z} / N \mathbb{Z}) \mathcal{O}(U(1))$ is a non-cleft $\mathcal{O}(U(1))$-comodule algebra.

Now we will try to describe the algebra $\mathcal{O}\left(S_{p q \theta}^{3}\right) \square_{\mathcal{O}(\mathbb{Z} / N \mathbb{Z})} \mathcal{O}(U(1))$ in more detail. First, we observe that the cotensor product $\mathcal{O}\left(S_{p q \theta}^{3}\right) \square_{\mathcal{O}(\mathbb{Z} / N \mathbb{Z})} \mathcal{O}(U(1))$ is equal to $\left(\mathcal{O}\left(S_{p q \theta}^{3}\right) \otimes \mathcal{O}(U(1))\right)^{\operatorname{co} \mathcal{O}(\mathbb{Z} / N \mathbb{Z})}$ for the coaction

$$
\begin{aligned}
& \mathcal{O}\left(S_{p q \theta}^{3}\right) \otimes \mathcal{O}(U(1)) \ni p \otimes h \\
& \longmapsto p_{(0)} \otimes h_{(2)} \otimes p_{(1)} S\left(\pi\left(h_{(1)}\right)\right) \in \mathcal{O}\left(S_{p q \theta}^{3}\right) \otimes \mathcal{O}(U(1)) \otimes \mathcal{O}(\mathbb{Z} / N \mathbb{Z}) .
\end{aligned}
$$

This coaction defines the following $\mathbb{Z} / N \mathbb{Z}$-action:

$$
\begin{aligned}
& \tilde{\alpha}: \mathbb{Z} / N \mathbb{Z} \ni e^{\frac{2 \pi i k}{N}} \longmapsto \tilde{\alpha}_{e^{\frac{2 \pi i k}{N}}} \in \operatorname{Aut}\left(\mathcal{O}\left(S_{p q \theta}^{3}\right) \otimes \mathcal{O}(U(1))\right), \\
& \tilde{\alpha}_{e^{\frac{2 \pi i k}{N}}}(x \otimes h):=\alpha_{e^{\frac{2 \pi i k}{N}}}(x) \otimes h\left(\cdot e^{\frac{-2 \pi i k}{N}}\right) .
\end{aligned}
$$

With this action in mind, we can now write

$$
\mathcal{O}\left(S_{p q \theta}^{3}\right) \underset{\mathcal{O}(\mathbb{Z} / N \mathbb{Z})}{\square} \mathcal{O}(U(1))=\left(\mathcal{O}\left(S_{p q \theta}^{3}\right) \otimes \mathcal{O}(U(1))\right)^{\mathbb{Z} / N \mathbb{Z}} .
$$

Next, a straightforward calculation inspired by [4, Lemma 5.3] and taking advantage of the Hopf $*$-algebra isomorphism

$$
\begin{aligned}
\psi: \mathcal{O}(U(1)) \ni u \longmapsto u^{N} \in & \operatorname{co} \mathcal{O}(\mathbb{Z} / N \mathbb{Z}) \\
: & \mathcal{O}(U(1)) \\
& :=\left\{h \in \mathcal{O}(U(1)) \mid \pi\left(h_{(1)}\right) \otimes h_{(2)}=1 \otimes h\right\}
\end{aligned}
$$

allows us to prove the following result. 
Proposition 3.2. The assignments

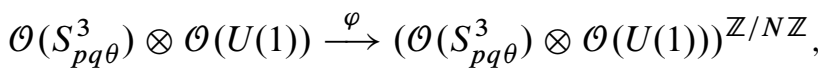

$$
\begin{aligned}
& x \otimes h \stackrel{\varphi}{\longmapsto} x_{(0)} \otimes x_{(1)} \psi(h), \\
& \left(\mathcal{O}\left(S_{p q \theta}^{3}\right) \otimes \mathcal{O}(U(1))\right)^{\mathbb{Z} / N \mathbb{Z}} \stackrel{\varphi^{-1}}{\longrightarrow} \mathcal{O}\left(S_{p q \theta}^{3}\right) \otimes \mathcal{O}(U(1)), \\
& \sum_{i} x^{i} \otimes h^{i} \stackrel{\varphi^{-1}}{\longmapsto} \sum_{i} x_{(0)}^{i} \otimes \psi^{-1}\left(S\left(x_{(1)}^{i}\right) h^{i}\right),
\end{aligned}
$$

define mutually inverse isomorphisms of $*$-algebras.

3.2. Prolongations in the $\boldsymbol{C}^{*}$-setting. As above, we can argue that the prolongation of $C\left(S_{p q \theta}^{3}\right)$ by $\mathcal{O}(U(1))$ is a principal comodule algebra. However, we need to apply a different reasoning than above to show that it is not cleft. Recall first that, by Theorem 2.2, the finitely generated projective left $C\left(L_{p q \theta}^{N}\right)$-module $L_{N}$ is not free. Together with the natural identifications

$$
L_{N} \cong C\left(S_{p q \theta}^{3}\right)_{\mathcal{O}(\mathbb{Z} / N \mathbb{Z})}^{\square} \mathbb{C} \cong C\left(S_{p q \theta}^{3}\right) \underset{\mathcal{O}(\mathbb{Z} / N \mathbb{Z})}{\square} \mathcal{O}(U(1)) \underset{\mathcal{O}(U(1))}{\square} \mathbb{C}
$$

we see that the rightmost module is also not free. Since every module associated with a cleft comodule algebra is necessarily free, we arrive at the following corollary of Theorem 2.2.

Corollary 3.3. The $\mathcal{O}(U(1))$-comodule algebra $C\left(S_{p q \theta}^{3}\right) \underset{\mathcal{O}(\mathbb{Z} / N \mathbb{Z})}{\square} \mathcal{O}(U(1))$ is not cleft.

To prove an analogue of Proposition 3.2, we use the identification $C(X, A) \cong$ $A \bar{\otimes} C(X)$, where $X$ is a compact Hausdorff space, $A$ is a unital $C^{*}$-algebra, $C(X, A)$ is the algebra of norm-continuous functions, and $C(X):=C(X, \mathbb{C})$. Furthermore, we easily check that the formulae

$$
\begin{aligned}
\left(F_{1}(f)\right)\left(e^{i \varphi_{1}}, e^{i \varphi_{2}}\right) & :=\alpha_{e^{i \varphi_{1}}}\left(f\left(e^{i \varphi_{2}}\right)\right), \\
\left(F_{2}(g)\right)\left(e^{i \varphi}\right) & :=g\left(e^{i \varphi}, e^{i \varphi N}\right), \\
\left(G_{1}(f)\right)\left(e^{i \varphi_{1}}, e^{i \varphi_{2}}\right) & :=\alpha_{e^{i \varphi_{1}}}\left(f\left(e^{i \varphi_{2}}\right)\right), \\
\left(G_{2}(g)\right)\left(e^{i \varphi}\right) & :=g\left(e^{\frac{-i \varphi}{N}}, e^{\frac{i \varphi}{N}}\right),
\end{aligned}
$$


define $C^{*}$-homomorphisms in the diagram

$$
\begin{gathered}
C\left(U(1), C\left(S_{p q \theta}^{3}\right)\right) \underset{G_{2}}{\stackrel{F_{1}}{\gtrless}} C\left(U(1) \times U(1), C\left(S_{p q \theta}^{3}\right)\right) \underset{G_{1}}{\stackrel{F_{2}}{\rightleftarrows}} C_{\mathbb{Z} / N \mathbb{Z}}\left(U(1), C\left(S_{p q \theta}^{3}\right)\right) \\
\uparrow \cong \\
C\left(S_{p q \theta}^{3}\right) \bar{\otimes} C(U(1)) \underset{\uparrow}{\rightleftarrows}\left(C\left(S_{p q \theta}^{3}\right) \bar{\otimes} C(U(1))\right)^{\mathbb{Z} / N \mathbb{Z}} .
\end{gathered}
$$

Here the right bottom corner is defined via an extension of the action (3.2) to the $C^{*}$-algebra $C\left(S_{p q \theta}^{3}\right) \bar{\otimes} C(U(1))$. Verifying that $F_{2} \circ F_{1}$ and $G_{2} \circ G_{1}$ are mutually inverse maps yields the desired isomorphism result.

Proposition 3.4. The $C^{*}$-algebras $\left(C\left(S_{p q \theta}^{3}\right) \bar{\otimes} C(U(1))\right)^{\mathbb{Z} / N \mathbb{Z}}$ and $C\left(S_{p q \theta}^{3}\right) \bar{\otimes} C(U(1))$ are isomorphic.

Acknowledgements. This work is part of the EU-project Geometry and symmetry of quantum spaces PIRSES-GA-2008-230836. It was partially supported by the Polish Government grant 1261/7.PRUE/2009/7. The first author gratefully acknowledges the generous hospitality of the Mathematical Sciences Institute of the Australian National University that made his visit to Canberra possible and productive. The first author is very grateful to Nigel Higson for clarifying to him the odd-to-even connecting homomorphism in the $C^{*}$-setting. The second author was supported by the Australian Research Council and the Australian Academy of Sciences. The third author thanks Tomasz Brzeziński for discussions.

\section{References}

[1] P. F. Baum and R. Meyer, The Baum-Connes conjecture, localisation of categories and quantum groups. In Lecture notes on noncommutative geometry and quantum groups, P. M. Hajac (ed.), EMS Publishing House, to appear.

[2] P. F. Baum, P. M. Hajac, R. Matthes, and W. Szymański, The $K$-theory of Heegaardtype quantum 3-spheres. K-Theory 35 (2005), 159-186; erratum ibid. 37 (2006), 211. Zbl 1210.46054 Zbl 1111.46051 MR 2240219 MR 2240219

[3] T. Brzeziński and P. M. Hajac, The Chern-Galois character. C. R. Math. Acad. Sci. Paris 338 (2004), 113-116. Zbl 1061.16037 MR 2038278

[4] T. Brzeziński and B. Zieliński, Quantum principal bundles over quantum real projective spaces. J. Geom. Phys. 62 (2012), 1097-1107. Zbl 1244.81033 MR 2901849

[5] L. Dạbrowski, H. Grosse, and P. M. Hajac, Strong connections and Chern-Connes pairing in the Hopf-Galois theory. Comm. Math. Phys. 220 (2001), 301-331. Zbl 0990.58008 MR 1844628 
[6] L. Dạbrowski, T. Hadfield, P. M. Hajac, R. Matthes, and E. Wagner, Index pairings for pullbacks of $\mathrm{C}^{*}$-algebras. In Operator algebras and quantum groups, Banach Center Publ. 98, Polish Acad. Sci. Inst. Math., Warsaw 2012, 67-84. Zbl 06137288 MR 3059658

[7] E. Guentner and N. Higson, Group $C^{*}$-algebras and $K$-theory. In Noncommutative geometry, Lecture Notes in Math. 1831, Springer, Berlin 2004, 137-251. Zbl 1053.46048 MR 2058474

[8] P. M. Hajac, Strong connections on quantum principal bundles. Comm. Math. Phys. 182 (1996), 579-617. Zbl 0873.58007 MR 1461943

[9] P. M. Hajac, U. Krähmer, R. Matthes, and B. Zieliński, Piecewise principal comodule algebras. J. Noncommut. Geom. 5 (2011), 591-614. Zbl 1258.16036 MR 2838527

[10] P. M. Hajac and S. Majid, Projective module description of the $q$-monopole. Comm. Math. Phys. 206 (1999), 247-264. Zbl 0936.46052 MR 1722149

[11] P. M. Hajac, R. Matthes, and W. Szymański, A locally trivial quantum Hopf fibration. Algebr. Represent. Theory 9 (2006), 121-146. Zbl 1132.58007 MR 2238363

[12] P. M. Hajac, R. Matthes, P. M. Sołtan, W. Szymański, and B. Zieliński, Hopf-Galois Extensions and $\mathrm{C}^{*}$-algebras. In Quantum symmetry in noncommutative geometry, P. M. Hajac (ed.), EMS Publishing House, to appear.

[13] P. M. Hajac, R. Matthes, and W. Szymański, Noncommutative index theory for mirror quantum spheres. C. R. Math. Acad. Sci. Paris 343 (2006), 731-736. Zbl 1114.46052 MR 2284701

[14] J. H. Hong and W. Szymański, Quantum lens spaces and graph algebras. Pacific J. Math. 211 (2003), 249-263. Zbl 1058.46047 MR 2015735

[15] S. Klimek and A. Lesniewski, A two-parameter quantum deformation of the unit disc. $J$. Funct. Anal. 115 (1993), 1-23. Zbl 0780.58020 MR 1228139

[16] J. Milnor, Introduction to algebraic K-theory. Ann. of Math. Stud. 72, Princeton University Press, Princeton, N.J., 1971. Zbl 0237.18005 MR 0349811

[17] J. H. Przytycki and A. Yasukhara, Symmetry of links and classification of lens spaces. Geom. Dedicata 98 (2003), 57-61. Zbl 1028.57018 MR 1988423

[18] C. Schochet, Topological methods for $C^{*}$-algebras. III. Axiomatic homology. Pacific J. Math. 114 (1984), 399-445. Zbl 0491.46061 MR 757510

[19] B. Zieliński Locally coalgebra-Galois extensions. Preprint 2005. arXiv:math/0512150

Received December 31, 2011

P. M. Hajac, Instytut Matematyczny, Polska Akademia Nauk, ul. Śniadeckich 8, 00-956 Warszawa, Poland, and Katedra Metod Matematycznych Fizyki, Uniwersytet Warszawski, ul. Hoża 74, 00-682 Warszawa, Poland

E-mail:pmh@impan.pl

A. Rennie, School of Mathematics and Applied Statistics, University of Wollongong, Wollongong, NSW 2522, Australia

E-mail: renniea@uow.edu.au

B. Zieliński, Department of Theoretical Physics and Computer Science, Faculty of Physics and Applied Informatics, University of Łódź, Pomorska 149/153, 90-236 Łódź, Poland

E-mail: bzielinski@uni.lodz.pl 